\title{
Hamiltonian 2-forms in Kähler geometry, IV Weakly Bochner-flat Kähler manifolds
}

\author{
Vestislav Apostolov, David M. J. Calderbank, Paul Gauduchon \\ and Christina W. Tønnesen-Friedman
}

\begin{abstract}
We study the construction and classification of weakly Bochnerflat (WBF) metrics (i.e., Kähler metrics with coclosed Bochner tensor) on compact complex manifolds. A Kähler metric is WBF if and only if its 'normalized' Ricci form is a hamiltonian 2-form: such 2-forms were introduced and studied in previous papers in the series. It follows that WBF Kähler metrics are extremal. We construct many new examples of WBF metrics on projective bundles and obtain a classification of compact WBF Kähler 6-manifolds, extending work by the first three authors on weakly selfdual Kähler 4-manifolds. The constructions are independent of previous papers in the series, but the classification relies on the classification of compact Kähler manifolds with a hamiltonian 2-form [3].
\end{abstract}

1 Introduction $\quad 92$

2 Hamiltonian 2-forms and WBF Kähler metrics 94

2.1 Classification of hamiltonian 2-forms 94

$\begin{array}{ll}2.2 & \text { Admissible bundles and metrics }\end{array}$

2.3 WBF Kähler metrics of order 0 and $1 \quad 97$

3 Kähler-Einstein metrics and Kähler-Ricci solitons 98

4 Constructions of WBF Kähler metrics 101

4.1 WBF Kähler metrics over a Kähler-Einstein manifold 
4.2 WBF Kähler metrics over a product of Kähler-Einstein manifolds

4.3 WBF Kähler metrics over a ruled surface

4.4 WBF versus extremal Kähler metrics

5 Classification of WBF Kähler metrics on compact 6-manifolds

Appendix. Proofs of Lemmas 4.3, 4.4 and 4.5

Acknowledgements

References

\section{Introduction}

A Kähler metric is said to be weakly Bochner-flat (WBF) if the Bochner tensor (a component of the curvature tensor) is coclosed. By the differential Bianchi identity, this is equivalent to an overdetermined first-order linear equation on the Ricci form $\rho$. Examples include Bochner-flat Kähler metrics (where the Bochner tensor is zero, see [6] for a classification) - in particular metrics of constant holomorphic sectional curvature (CHSC) and products of Kähler-Einstein metrics (for which $\rho$ is parallel).

The equation satisfied by the Ricci form of a WBF Kähler metric means that the normalized Ricci form $\tilde{\rho}:=\rho-\frac{\text { Scal }_{g}}{2(m+1)} \omega$ is a hamiltonian 2-form. Recall that a real $(1,1)$-form (i.e., a $J$-invariant 2 -form) $\phi$ on a Kähler manifold $(M, J, g, \omega)$, of real dimension $2 m>2$ is said to be hamiltonian [2] if

$$
2 \nabla_{X} \phi=d \operatorname{tr} \phi \wedge(J X)^{b}-(J d \operatorname{tr} \phi) \wedge X^{b}
$$

for all $X \in T M$ (where $X^{b}(Y)=g(X, Y)$ for $Y \in T M$ and $\left.\operatorname{tr} \phi=\langle\omega, \phi\rangle_{g}\right)$.

The momentum polynomial of a hamiltonian 2 -form $\phi$ is

$$
p(t):=(-1)^{m} \operatorname{pf}(\phi-t \omega)=t^{m}-(\operatorname{tr} \phi) t^{m-1}+\cdots+(-1)^{m} \operatorname{pf} \phi
$$

where the pfaffian is defined by $\phi \wedge \cdots \wedge \phi=(\operatorname{pf} \phi) \omega \wedge \cdots \wedge \omega$. The reason for calling $\phi$ hamiltonian is that the functions $p(t)$ on $M$ (for $t \in \mathbb{R}$ ) 
are Poisson-commuting hamiltonians for Killing vector fields $K(t):=J \operatorname{grad}_{g}$ $p(t)[2]$. The integer $\ell=\max _{x \in M} \operatorname{dim} \operatorname{span}\left\{K(t)_{x}: t \in \mathbb{R}\right\}$ is called the order of the hamiltonian 2-form (and $0 \leq \ell \leq m$ ). The order of a WBF metric is defined to be the order of its normalized Ricci form. Note that the FubiniStudy metric on $\mathbb{C} P^{m}$ has order zero, but admits hamiltonian 2-forms of any order $0 \leq \ell \leq m[2]$.

It follows that WBF Kähler metrics are extremal in the sense of [7]. We thus have the following implications between classes of Kähler metrics:

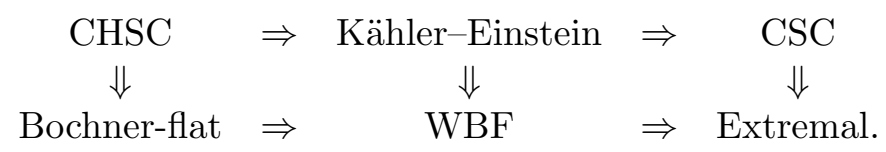

The observation that a Kähler metric is WBF if and only if the normalized Ricci form is hamiltonian motivated us to indulge in a detailed study of the local and global theory of hamiltonian 2-forms on Kähler manifolds [2,3], as well as the application of this to the theory of extremal Kähler metrics [4]. For the final paper in this series, we are now returning to our initial interest in WBF Kähler metrics.

We do not wish to impose the study of hamiltonian 2-forms on the reader of this paper, so we therefore propose to make the constructions of WBF metrics herein essentially self-contained, whereas for the necessity of the form of these constructions (both as motivation and as the source of the classification results we obtain) we review in Section 2 the facts we require from the general theory. These results will allow us to classify WBF metrics on compact 6 -manifolds.

The structure of the paper is as follows. In Section 2 we review the general theory of Kähler metrics with hamiltonian 2-forms [2-4] with a special attention to the case when the hamiltonian form has order $\ell=1$. We present an explicit construction of such metrics on a class of 'admissible' projective bundles of the form $M=P\left(E_{0} \oplus E_{\infty}\right) \rightarrow S$, where $E_{0}$ and $E_{\infty}$ are projectively flat hermitian vector bundles over a Kähler manifold $S$ endowed with compatible local product structure. According to [3,4], any Kähler manifold admitting a hamiltonian 2-form of order 1 is obtained by this construction up to a covering, and if there is no torsion in $H^{2}\left(S, \mathcal{O}^{*}\right)$, we can take the covering to be trivial.

In Section 3, as a warm-up, we use Kähler-Ricci solitons [11] to study Kähler-Einstein metrics on admissible bundles $M=P\left(E_{0} \oplus E_{\infty}\right) \rightarrow S$ where $S$ is a product of positive Kähler-Einstein manifolds. We show that a Kähler-Ricci soliton exists (and is unique) if and only if $M$ is a Fano 
manifold. These examples were found by Koiso [11], and the vanishing of the Futaki invariant is necessary and sufficient for the existence of a KählerEinstein metric, cf. [11].

In the remainder of the paper, we study WBF metrics in general. In Section 4 we construct many compact WBF manifolds of order 1, including all such examples in dimension 6. This leads to a classification of WBF 6-manifolds $M$ in Section 5: they are either order 0 and generalized Kähler-Einstein, or they are order 1, and - apart from one example on $P\left(\mathcal{O} \oplus \mathcal{O}(1) \otimes \mathbb{C}^{2}\right) \rightarrow \mathbb{C} P^{1}$ - are then projective line bundles over a ruled surface or a positive Kähler-Einstein surface. In each case the WBF Kähler metric is unique up to scale and pullback by an automorphism of $(M, J)$.

This is much richer than the classification of WBF 4-manifolds, where the only example of order 1 is the first Hirzebruch surface $P(\mathcal{O} \oplus \mathcal{O}(1)) \rightarrow$ $\mathbb{C} P^{1}[1]$. It is natural to conjecture that all compact WBF Kähler manifolds have order 0 or 1 , but such a result is out of reach using the explicit methods of this paper.

\section{Hamiltonian 2-forms and WBF Kähler metrics}

We begin by recalling the classification of compact Kähler manifolds with a hamiltonian 2-form from [2-4], focussing on the case that the hamiltonian 2 -form has order 1 . The output of this classification is a self-contained Ansatz that we shall use to construct WBF Kähler metrics in Section 4, so that we only need the results of [2-4] for the classification results we obtain. We adopt the notations and conventions of [4] and refer to [4, $\$ 1$ and App. A] for further information.

\subsection{Classification of hamiltonian 2-forms}

Let $(M, g, J, \omega)$ be a compact connected Kähler $2 m$-manifold with a hamiltonian 2-form $\phi$ of order $\ell$. Then, according to [3], the vector fields $\{K(t): t \in$ $\mathbb{R}\}$ described in the introduction generate an effective isometric hamiltonian action of an $\ell$-torus $\mathbb{T}$ on $M$. The stable quotient $\hat{S}$ of $M$ by the induced action of the complexified torus $\mathbb{T}^{c}$ is covered by a product of Kähler manifolds $S_{a}$ indexed by the distinct constant roots of $p(t)$, the dimension of $S_{a}$ being $2 d_{a}$, where $d_{a}$ is the multiplicity of the corresponding root.

It was also shown in $[3,4]$ that there is a subset $\mathcal{A}$ of the constant roots such that $M$ is a projective bundle, over a complex manifold $S$ covered by $\prod_{a \in \mathcal{A}} S_{a}$, in such a way that $\hat{S}$ is a fibre product of flat projective unitary 
bundles over $S$, indexed by the remaining constant roots. In this paper, we shall always be in a situation where the following assumption holds for these bundles.

Assumption 2.1. A flat projective unitary $\mathbb{C} P^{r}$-bundle on $S$ is of the form $P(E)$, where $E$ is a rank $r+1$ projectively flat hermitian holomorphic vector bundle.

If $S$ is simply connected, then any flat projective unitary $\mathbb{C} P^{r}$-bundle is trivial, hence of the form $P(E)$ with $E \cong \mathcal{E} \otimes \mathbb{C}^{r+1}$ for a holomorphic line bundle $\mathcal{E}$. In general the obstruction to the existence of $E$ is given by a torsion element of $H^{2}\left(S, \mathcal{O}^{*}\right)$ (cf. [8]). In particular, such an $E$ always exists if $S$ is a Riemann surface.

It then follows, as in [4, App. A], that by formally adjoining additional constant roots of multiplicity 0 (corresponding to $\mathbb{C} P^{0}$ bundles over $S$ ) that we can write $\hat{S}=P\left(E_{0}\right) \times{ }_{S} P\left(E_{1}\right) \times{ }_{S} \cdots \times{ }_{S} P\left(E_{\ell}\right) \rightarrow S$, where $E_{j} \rightarrow$ $S$ are projectively flat hermitian bundles of ranks $d_{j}+1\left(d_{j} \geq 0\right)$, which can be chosen so that $M=P\left(E_{0} \oplus E_{1} \cdots \oplus E_{\ell}\right) \rightarrow S$. Thus the distinct constant roots are labelled by $\hat{\mathcal{A}}:=\mathcal{A} \cup\{0,1, \ldots \ell\}$, and $S_{a} \cong \mathbb{C} P^{d_{a}}$ for $a \in$ $\{0,1, \ldots \ell\}$. We remark that $M$ has a blow-up of the form $\hat{M}=P\left(\mathcal{L}_{0} \oplus\right.$ $\left.\mathcal{L}_{1} \cdots \oplus \mathcal{L}_{\ell}\right) \rightarrow \hat{S}$ for line bundles $\mathcal{L}_{j}$. If $d_{j}=0$ for all $j \in\{0,1, \ldots \ell\}$ then $\hat{M}=M$ and $\hat{S}=S$. Otherwise we say a blow-down occurs.

The extreme cases $\ell=0$ and $\ell=m$ are quite straightforward.

- If $\ell=0, M=\hat{S}=S$ is a local Kähler product and the hamiltonian 2 -form $\phi$ is a constant linear combination of the corresponding Kähler forms.

- If $\ell=m,(M, J)$ is biholomorphic to $\mathbb{C} P^{m}$ (and $\hat{S}=S$ is a point).

For the intermediate cases, there is also an explicit description, but we shall only need it in the case $\ell=1$ to which we now turn. Here it is convenient to index the constant roots by $\hat{\mathcal{A}}=\mathcal{A} \cup\{0, \infty\}$ so that $\mathcal{A}$ can be taken as a finite subset of $\mathbb{Z}^{+}$.

\subsection{Admissible bundles and metrics}

Definition 2.1. A projective bundle of the form $M=P\left(E_{0} \oplus E_{\infty}\right) \stackrel{p}{\rightarrow} S$ will be called admissible or an admissible manifold if:

- $S$ is a covered by a product $\tilde{S}=\prod_{a \in \mathcal{A}} S_{a}$ (for $\mathcal{A} \subset \mathbb{Z}^{+}$) of simplyconnected Kähler manifolds $\left(S_{a}, \pm g_{a}, \pm \omega_{a}\right)$ of real dimensions $2 d_{a}$; 
- $E_{0}$ and $E_{\infty}$ are holomorphic projectively flat hermitian vector bundles over $S$ of ranks $d_{0}+1$ and $d_{\infty}+1$ with $\bar{c}_{1}\left(E_{\infty}\right)-\bar{c}_{1}\left(E_{0}\right)=\left[\omega_{S} / 2 \pi\right]$ and $\omega_{S}=\sum_{a \in \mathcal{A}} \omega_{a}$, where $\bar{c}_{1}(E)=c_{1}(E) / \operatorname{rank} E$.

In the first condition, it is convenient to let $\left(g_{a}, \omega_{a}\right)$ be positive or negative definite: otherwise we would have to admit signs in the definition of $\omega_{S}$. The second condition means that we can fix hermitian metrics on $E_{0}$ and $E_{\infty}$ whose Chern connections have trace-like curvatures $\Omega_{0} \otimes I d_{E_{0}}$ and $\Omega_{\infty} \otimes$ $I d_{E_{\infty}}$ satisfying $\Omega_{\infty}-\Omega_{0}=\omega_{S}$. We normalize the induced fibrewise FubiniStudy metrics $\left(g_{0}, \omega_{0}\right)$ and $\left(-g_{\infty},-\omega_{\infty}\right)$ on $P\left(E_{0}\right)$ and $P\left(E_{\infty}\right)$ to have scalar curvatures $2 d_{0}\left(d_{0}+1\right)$ and $2 d_{\infty}\left(d_{\infty}+1\right)$.

We also have $\hat{M}=P(\mathcal{O} \oplus \hat{\mathcal{L}}) \rightarrow \hat{S} \quad$ with $\quad c_{1}(\hat{\mathcal{L}})=\left[\omega_{\hat{S}} / 2 \pi\right] \quad$ and $\omega_{\hat{S}}=\sum_{a \in \hat{\mathcal{A}}} \omega_{a}$.

Remark 2.1. The existence of the line bundle $\hat{\mathcal{L}} \rightarrow \hat{S}$ with $c_{1}(\hat{\mathcal{L}})=\left[\omega_{\hat{S}} / 2 \pi\right]$ implies that $\omega_{\hat{S}}$ is integral in the sense that $\left[\omega_{\hat{S}} / 2 \pi\right]$ is in the image of $H^{2}(\hat{S}, \mathbb{Z})$ in $H^{2}(\hat{S}, \mathbb{R})$. When $\hat{S}$ is a global Kähler product (so we have $\left.M=P\left(\mathcal{O} \otimes \mathbb{C}^{d_{0}+1} \oplus \mathcal{L} \otimes \mathbb{C}^{d_{\infty}+1}\right) \rightarrow S=\prod_{a \in \mathcal{A}} S_{a}\right)$, this integrality condition means that each $\omega_{a}$ is integral, i.e., the compact manifolds $\left(S_{a}, \pm g_{a}\right.$, $\left.\pm \omega_{a}\right)$ are Hodge. We write $\omega_{a}=q_{a} \alpha_{a}$ for an integer $q_{a} \neq 0$, where $\alpha_{a}$ is a primitive integral Kähler form on $S_{a}$, so that $q_{a}$ is a nonzero integer with the same sign as $\left(g_{a}, \omega_{a}\right)$, and $q_{0}=1$ and $q_{\infty}=-1$.

If $\pm g_{a}$ is Kähler-Einstein, then $\rho_{a}=p_{a} \alpha_{a}$ where $p_{a}$ is an integer (called the Fano index for positive Kähler-Einstein metrics). We set $s_{a}=p_{a} / q_{a}$ and then $\mathrm{Scal}_{a}= \pm 2 d_{a} s_{a}$, where the sign is that of $q_{a}$, so the scalar curvature of $\pm g_{a}$ has the same sign as $p_{a}$. For instance, if $S_{a}$ is $\mathbb{C} P^{1}$ and $g_{a}$ is negative definite (i.e., $q_{a}$ is negative), then $\mathrm{Scal}_{a}$ is positive (and $p_{a}$ is positive), but $s_{a}$ is negative. By the well-known Kobayashi-Ochiai inequality [10] $p_{a} \leq d_{a}+1$, where equality holds iff $S_{a}=\mathbb{C} P^{d_{a}}$. Comparing the Chern classes $c_{1}\left(\mathcal{L}_{a}\right)=\left[q_{a} \alpha_{a} / 2 \pi\right]$ and $c_{1}\left(\mathcal{K}^{-1}\right)=\left[p_{a} \alpha_{a} / 2 \pi\right]$, we have that $\mathcal{L}_{a}^{p_{a}}$ is $\mathcal{K}^{-q_{a}}$ tensored by a flat line bundle. If $p_{a}$ is not zero (i.e., $S_{a}$ is not Ricci-flat), this gives $\mathcal{L}_{a} \cong \mathcal{K}^{-q_{a} / p_{a}} \otimes \mathcal{L}_{a, 0}$ for some flat line bundle $\mathcal{L}_{a, 0}$. For instance if $S_{a}=\mathbb{C} P^{d_{a}}$, then $p_{a}=d_{a}+1$ and $\mathcal{L}_{a} \cong \mathcal{O}\left(q_{a}\right)$.

We now describe the Kähler metrics which admit a hamiltonian 2-form $\phi$ of order $\ell=1$. In this case the hamiltonian torus action is just an $S^{1}$ action generated by a single hamiltonian Killing vector field $K=J \operatorname{grad}_{g} z$, and without loss, we can take the image of its momentum map $z$ to be $[-1,1]$. We denote the constant roots by $-1 / x_{a}$ and we have that $0<$ $\left|x_{a}\right| \leq 1$ with equality iff $a \in\{0, \infty\}$; we can take $x_{0}=1$ and $x_{\infty}=-1$. 
Then $M^{0}:=z^{-1}((-1,1))$ is a principal $\mathbb{C}^{\times}$-bundle over $\hat{S}$ with connection 1 -form $\theta(\theta(K)=1)$ and there are Kähler metrics $\left( \pm g_{a}, \pm \omega_{a}\right)$, which are Fubini-Study metrics for $a \in\{0, \infty\}$, with the signs chosen so that $\omega_{a} / x_{a}$ is positive for all $a$, together with a smooth function $\Theta$ on $[-1,1]$ such that the Kähler structure on $M^{0}$ is

$$
\begin{aligned}
g & =\sum_{a \in \hat{\mathcal{A}}} \frac{1+x_{a} z}{x_{a}} g_{a}+\frac{d z^{2}}{\Theta(z)}+\Theta(z) \theta^{2} \\
\omega & =\sum_{a \in \hat{\mathcal{A}}} \frac{1+x_{a} z}{x_{a}} \omega_{a}+d z \wedge \theta, \quad \text { where } d \theta=\sum_{a \in \hat{\mathcal{A}}} \omega_{a},
\end{aligned}
$$

and $\Theta$ satisfies

$$
\begin{gathered}
\Theta>0 \quad \text { on } \quad(-1,1), \\
\Theta( \pm 1)=0, \quad \Theta^{\prime}( \pm 1)=\mp 2 .
\end{gathered}
$$

It follows from $[3,4]$ that if $M$ admits a hamiltonian 2-form of order 1 and either Assumption 2.1 holds or no blow-downs occur, then $M=P\left(E_{0} \oplus\right.$ $\left.E_{\infty}\right) \rightarrow S$ is an admissible bundle, and the above conditions are necessary and sufficient for the compactification of a metric of the form (2.1) on $M$, where $z: M \rightarrow[-1,1]$ with $P\left(E_{0} \oplus 0\right)=z^{-1}(1)$ and $P\left(0 \oplus E_{\infty}\right)=z^{-1}(-1)$, $\theta$ is a connection 1-form (see [4] for more details), the $S^{1}$ action generated by $K$ is given by scalar multiplication in $E_{\infty}$ (or equivalently in $E_{0}$ ), and the local product structure in (2.1) coincides with the given local product structure on $\hat{S}=P\left(E_{0}\right) \times_{S} P\left(E_{\infty}\right) \rightarrow S$.

We refer to a compatible metric of the form (2.1) on an admissible bundle as an admissible metric. It is straightforward (and standard) to see that the conditions (2.2) and (2.3) are sufficient for the compactification of metrics of the form, so that we can regard the above as an Ansatz for constructing Kähler metrics on admissible bundles, independently of the theory of hamiltonian 2-forms.

\subsection{WBF Kähler metrics of order 0 and 1}

According to the theory of hamiltonian 2-forms, a WBF Kähler manifold $M$ of order 0 is a local Kähler product and the normalized Ricci form is a constant linear combination of the corresponding Kähler forms. It follows that $M$ is generalized Kähler-Einstein (i.e., its universal cover is a product of Kähler-Einstein manifolds). 
In the order 1 case, we have the following characterization of WBF Kähler metrics of the form (2.1).

Proposition 2.1. Let $(g, J, \omega)$ be a Kähler metric with a hamiltonian 2-form $\phi$ of order 1 as in (2.1), and write $F(t)=\Theta(t) p_{c}(t)$ with $p_{c}(t)=\prod_{a \in \hat{\mathcal{A}}}$ $\left(1+x_{a} t\right)^{d_{a}}$. Then $g$ is $W B F$, with $\tilde{\rho}$ a constant linear combination of $\phi$ and $\omega$, iff

- $F^{\prime}(t)=Q(t) p_{c}(t)$ and $Q$ is a polynomial of degree $\leq 2$;

- for all $a, \pm g_{a}$ is Kähler-Einstein with scalar curvature $\pm d_{a} Q\left(-1 / x_{a}\right)$. $g$ is then Kähler-Einstein iff $Q$ has degree $\leq 1$.

(Here we use the conventions of [4], so that, compared with [2], we have $\eta_{a}=-1 / x_{a}$ and have rescaled $F(z)$ and $p_{c}(z)$ by $\left.\prod_{a \in \hat{\mathcal{A}}} x_{a}.\right)$

For the necessity of these conditions when $(g, J, \omega)$ is WBF, we refer to [2], but their sufficiency is a straightforward verification. Together with the discussion of the previous paragraph, we therefore have an Ansatz for constructing admissible WBF Kähler metrics on admissible projective bundles.

\section{Kähler-Einstein metrics and Kähler-Ricci solitons}

Recall that a Kähler-Ricci soliton on a compact complex manifold $(M, J)$ is a compatible Kähler metric $(g, \omega)$ satisfying

$$
\rho-\lambda \omega=\mathcal{L}_{V} \omega,
$$

where $V$ is a real holomorphic vector field with zeros and $\lambda$ is a real constant (necessarily equal to $\int_{M} \operatorname{Scal}_{g} \omega^{m} / \int_{M} \omega^{m}$ ). It follows from (3.1) that the Futaki invariant $\mathfrak{F}_{[\omega]}(V)$ vanishes iff the metric is Kähler-Einstein: if $V=$ $J \operatorname{grad}_{g} f+\operatorname{grad}_{g} h, \mathcal{L}_{V} \omega=d d^{c} h$ and the imaginary part of $\mathfrak{F}_{[\omega]}(V)$ reduces, after integrating by parts, to a nonzero multiple of the $L^{2}$-norm of $\operatorname{grad}_{g} h$; if this is zero, $V$ is a hamiltonian Killing vector field, so $\mathcal{L}_{V} \omega=0$. Note that if $V$ is nonzero then by the Bochner formula $\lambda>0$, and so $c_{1}(M)$ is positive, i.e., $(M, J)$ is a Fano manifold.

The theory of Kähler-Ricci solitons on Fano manifolds has recently received attention as a natural generalization of Kähler-Einstein metrics. In particular, a number of uniqueness results for such metrics have been established $[18,19]$, as well as existence results in the case of toric Fano manifolds [20] and certain geometrically ruled complex manifolds [11]. 
We now adapt arguments from [11] to construct (admissible) KählerRicci solitons on admissible projective bundles $M=P\left(\mathcal{O} \otimes \mathbb{C}^{d_{0}+1} \oplus\right.$ $\left.\mathcal{L} \otimes \mathbb{C}^{d_{\infty}+1}\right) \rightarrow S$, by taking $V=(c / 2) \operatorname{grad}_{g} z$ for a real constant $c$. Since $\mathcal{L}_{V} \omega=(c / 2) d d^{c} z$ and

$$
\rho=\sum_{a \in \hat{\mathcal{A}}} \rho_{a}-\frac{1}{2} d d^{c} \log F=\sum_{a \in \hat{\mathcal{A}}} \rho_{a}-\frac{1}{2} \frac{F^{\prime}(z)}{p_{\mathrm{c}}(z)} \sum_{a \in \hat{\mathcal{A}}} \omega_{a}-\frac{1}{2}\left(\frac{F^{\prime}}{p_{\mathrm{c}}}\right)^{\prime}(z) d z \wedge \theta
$$

where $F$ and $p_{c}$ are as defined in Proposition 2.1 (see [2]), (3.1) is equivalent to

$$
\begin{aligned}
\sum_{a \in \hat{\mathcal{A}}} \rho_{a}= & \sum_{a \in \hat{\mathcal{A}}} \frac{1}{2}\left(\frac{F^{\prime}(z)}{p_{\mathrm{c}}(z)}+c \frac{F(z)}{p_{\mathrm{c}}(z)}+2 \lambda\left(z+\frac{1}{x_{a}}\right)\right) \omega_{a} \\
& \left(\frac{F^{\prime}}{p_{\mathrm{c}}}\right)^{\prime}(z)+c\left(\frac{F}{p_{\mathrm{c}}}\right)^{\prime}(z)+2 \lambda=0 .
\end{aligned}
$$

Now (3.3) implies that for all $a,\left( \pm g_{a}, \pm \omega_{a}\right)$ is Kähler-Einstein and

$$
\frac{F^{\prime}(z)}{p_{\mathrm{c}}(z)}+c \frac{F(z)}{p_{\mathrm{c}}(z)}=2 s_{a}-2 \lambda\left(z+\frac{1}{x_{a}}\right) .
$$

Conversely this implies (3.3)-(3.4), the latter being just the derivative of (3.5).

As in $[4, \S 2.4]$, since $\Theta(z)=F(z) / p_{\mathrm{c}}(z)$, an application of l'Hôpital's rule shows that $(2.3)$ is equivalent to

$$
F( \pm 1)=0, \quad \Psi(-1)=2\left(d_{0}+1\right), \quad \Psi(1)=-2\left(d_{\infty}+1\right)
$$

where $F^{\prime}(z)=\Psi(z) p_{\mathrm{c}}(z)$. Hence evaluating (3.5) at $z= \pm 1$, we have

$$
\begin{aligned}
2 \lambda & =d_{0}+d_{\infty}+2 \\
2 s_{a} x_{a} & =\left(d_{\infty}+1\right)\left(1-x_{a}\right)+\left(d_{0}+1\right)\left(1+x_{a}\right),
\end{aligned}
$$

both expressions being manifestly positive (so the base manifolds $S_{a}$ have positive scalar curvature). These equations allow us to rewrite (3.5) as a single equation

$$
\frac{F^{\prime}(z)}{p_{\mathrm{c}}(z)}+c \frac{F(z)}{p_{\mathrm{c}}(z)}=\left(d_{0}+1\right)(1-z)-\left(d_{\infty}+1\right)(1+z)
$$


and (3.8) and (3.9) imply (3.5). Using (3.9), the boundary conditions (3.6) reduce to

$$
F( \pm 1)=0
$$

Hence we must solve (3.8)-(3.10) subject to $0<\left|x_{a}\right|<1$ and $F(z)>0$ for $z \in(-1,1)$. Clearly $(3.8)$ gives $x_{a}=\left(d_{0}+d_{\infty}+2\right) /\left(2 s_{a}+d_{\infty}-d_{0}\right)$ and so we must have

$$
\begin{aligned}
& s_{a}>d_{0}+1 \quad \text { if } \omega_{a}>0, \\
& s_{a}<-\left(d_{\infty}+1\right) \quad \text { if } \omega_{a}<0 .
\end{aligned}
$$

Restricting the formula (3.2) for $\rho$ to the zero and infinity sections $e_{0}$ and $e_{\infty}$, we see that these are actually necessary conditions for $c_{1}(M)=[\rho / 2 \pi]$ to be positive.

We now observe that

$$
F(z)=e^{-c z} \int_{-1}^{z} e^{c t}\left(\left(d_{0}+1\right)(1-t)-\left(d_{\infty}+1\right)(1+t)\right) p_{\mathrm{c}}(t) d t
$$

solves (3.9) and (3.10) iff $G(c)=0$, where

$$
\begin{aligned}
G(k) & =\int_{-1}^{1} e^{k t}\left(\left(d_{0}+1\right)(1-t)-\left(d_{\infty}+1\right)(1+t)\right) p_{\mathrm{c}}(t) d t \\
& =e^{k t_{0}} \int_{-1}^{1} e^{k\left(t-t_{0}\right)}\left(t-t_{0}\right) g(t) d t
\end{aligned}
$$

for some $t_{0} \in(-1,1)$ and $g(t)$ with $g<0$ on $(-1,1)$. Clearly $e^{-k t_{0}} G(k)$ is a strictly decreasing function of $k$ tending to $\mp \infty$ as $k \rightarrow \pm \infty$, so it has a unique zero $c$ (consistent with the uniqueness of Ricci solitons). Since $F^{\prime}$ has exactly one zero (namely $t_{0}$ ) in $(-1,1), F( \pm 1)=0$ and $F$ is positive near the endpoints, it is positive on $(-1,1)$. We deduce the following equivalence, essentially due to Koiso [11].

Theorem 3.1. Let $S=\prod_{a \in \mathcal{A}} S_{a}$ be a finite product $\left(\mathcal{A} \subset \mathbb{Z}^{+}\right)$of compact Kähler-Einstein manifolds $\left(S_{a}, \pm g_{a}, \pm \omega_{a}\right)$ with scalar curvatures $\mathrm{Scal}_{a}=$ $\pm 2 d_{a} s_{a}$ and let $M=P\left(\mathcal{O} \otimes \mathbb{C}^{d_{0}+1} \oplus \mathcal{L} \otimes \mathbb{C}^{d_{\infty}+1}\right) \rightarrow S$, where $\mathcal{L}=\bigotimes_{a \in \mathcal{A}} \mathcal{L}_{a}$ and $\mathcal{L}_{a}$ are line bundles over $S_{a}$ with $c_{1}\left(\mathcal{L}_{a}\right)=\left[\omega_{a} / 2 \pi\right]$. Then the following conditions are equivalent:

- the conditions (3.11) and (3.12) are satisfied; 
- $(M, J)$ is a Fano manifold;

- there exists a Kähler-Ricci soliton on $(M, J)$.

In this case, the Kähler-Ricci soliton $(g, \omega)$ is admissible with $\lambda=\left(d_{0}+\right.$ $\left.d_{\infty}+1\right) / 2$ and $V=(c / 2) \operatorname{grad}_{g} z$ for a suitable real constant $c$.

Our arguments and the fact that any Fano manifold is simply connected show that Theorem 3.1 gives all compact Kähler-Ricci solitons compatible with a hamiltonian 2 -form of order 1 as above. We also have the following standard corollary.

Corollary 3.1. [11] Let $M^{2 m}=P\left(\mathcal{O} \otimes \mathbb{C}^{d_{0}+1} \oplus \mathcal{L} \otimes \mathbb{C}^{d_{\infty}+1}\right) \rightarrow S$, as in the above theorem. Then there is a Kähler-Einstein metric on $M$ if and only if the conditions (3.11) and (3.12) are satisfied and the Futaki invariant $\mathfrak{F}_{[\rho]}(K)$ vanishes.

The Futaki invariant $\mathfrak{F}_{[\rho]}(K)$ is a nonzero multiple of the coefficient of $z^{m+2}$ in the extremal polynomial $F_{[\rho]}(z)$ as defined in [4] (which is the leading coefficient if it is nonzero). Hence its vanishing is equivalent to $F_{[\rho]}$ having degree at most $m+1$. Unfortunately, verifying this condition is not easy (it leads to a nontrivial diophantine problem); we will rediscover some KählerEinstein examples of $[12,13]$ in the next section as a byproduct of our study of WBF metrics.

\section{Constructions of WBF Kähler metrics}

We turn now to the construction of admissible WBF Kähler metrics on admissible projective bundles. By Proposition 2.1, an admissible metric $g$ with $F(z)=\Theta(z) p_{\mathrm{c}}(z), p_{\mathrm{c}}(z)=\prod_{a}\left(1+x_{a} z\right)^{d_{a}}\left(0 \leq a \leq \infty, d_{a} \geq 0\right)$ is WBF, with $\tilde{\rho}$ a linear combination of the hamiltonian 2 -form $\phi$ and the Kähler form $\omega$, precisely when the metrics $g_{a}$ are Kähler-Einstein and

$$
F^{\prime}(z)=p_{\mathrm{c}}(z) Q(z)
$$

for a polynomial $Q$ of degree $\leq 2$ with

$$
Q\left(-1 / x_{a}\right)=2 s_{a} \quad(a \in \hat{\mathcal{A}}) .
$$

In this case $F$ is the extremal polynomial of the corresponding admissible Kähler class [4] and the WBF Kähler metric is Kähler-Einstein iff $Q$ has degree $\leq 1$. 
Since $g$ is, in particular, extremal, we know from [4] (and it is straightforward to check) that the positivity (2.2) and endpoint conditions (2.3) may be replaced with

$$
\begin{aligned}
F>0 \quad \text { on } \quad(-1,1) \\
F( \pm 1)=0, \quad F^{\prime}( \pm 1)=\mp 2 p_{\mathrm{c}}( \pm 1) .
\end{aligned}
$$

Using equations (4.1) and (4.2), equation (4.4) implies that $Q(-1)=$ $2\left(d_{0}+1\right)$ and $Q(1)=-2\left(d_{\infty}+1\right)$. We remark that since $Q(z)$ therefore changes sign only once on $(-1,1)$, so does $F^{\prime}(z)$ (since $p_{\mathrm{c}}(z)$ is positive). Hence $F(z)$ (and $F(z) / p_{\mathrm{c}}(z)$ ) will be positive on $(-1,1)$ as soon as $(4.4)$ is satisfied.

The general quadratic $Q$ satisfying $Q(-1)=2\left(d_{0}+1\right)$ and $Q(1)=-2$ $\left(d_{\infty}+1\right)$ is

$$
Q(z)=B\left(1-z^{2}\right)+\left(d_{0}+1\right)(1-z)-\left(d_{\infty}+1\right)(1+z)
$$

(and the Kähler-Einstein case is when $B=0$ ). Equation (4.2) gives

$$
2 s_{a} x_{a}^{2}=B\left(x_{a}^{2}-1\right)+\left(d_{0}+1\right)\left(1+x_{a}\right) x_{a}+\left(d_{\infty}+1\right)\left(1-x_{a}\right) x_{a} .
$$

We write $B=B_{a}$ for the solutions of these equations $(a \in \mathcal{A})$, so that

$$
B_{a}:=\frac{x_{a}\left(\left(d_{0}+1\right)\left(1+x_{a}\right)+\left(d_{\infty}+1\right)\left(1-x_{a}\right)-2 s_{a} x_{a}\right)}{\left(1-x_{a}^{2}\right)} .
$$

On the other hand, given the above, then (4.4) is satisfied iff we set $F(z)=\int_{-1}^{z} p_{\mathrm{c}}(t)\left(B\left(1-t^{2}\right)+\left(d_{0}+1\right)(1-t)-\left(d_{\infty}+1\right)(1+t)\right) d t$ and

$$
\int_{-1}^{1} p_{\mathrm{c}}(t)\left(B\left(1-t^{2}\right)+\left(d_{0}+1\right)(1-t)-\left(d_{\infty}+1\right)(1+t)\right) d t=0 .
$$

Since $p_{\mathrm{c}}(t)\left(1-t^{2}\right)$ is positive on $(-1,1)$, this determines $B$ uniquely, once all other quantities are known. Hence, in order to complete the construction, we must show that $B=B_{a}$ solves (4.7) for all $a \in \mathcal{A}$. Multiplying by $1-x_{a}^{2}$, this means that $h_{a}=0$ for all such $a$, where

$$
\begin{aligned}
h_{a}= & \int_{-1}^{1} p_{\mathrm{c}}(t)\left(\left(1-x_{a}^{2}\right)\left(\left(d_{0}+1\right)(1-t)-\left(d_{\infty}+1\right)(1+t)\right)\right. \\
& \left.+x_{a}\left(\left(d_{0}+1\right)\left(1+x_{a}\right)+\left(d_{\infty}+1\right)\left(1-x_{a}\right)-2 s_{a} x_{a}\right)\left(1-t^{2}\right)\right) d t
\end{aligned}
$$


Our strategy for solving this problem is to use the equations $\left\{h_{a}=0\right.$ : $a \in \mathcal{A}\}$ to determine $\left\{x_{a}: a \in \mathcal{A}\right\}$ as functions of $\left\{s_{a}: a \in \mathcal{A}\right\}$. For given $s_{a}=p_{a} / q_{a}$, we obtain a WBF Kähler metric on the corresponding projective bundle iff we can find solutions $x_{a}$ with $0<\left|x_{a}\right|<1$. We note that $h_{a}=\int_{-1}^{1} p_{\mathrm{c}}(t) k_{a}(t) d t$, where

$$
\begin{aligned}
k_{a}(t)= & \left(\left(d_{0}+1\right)\left(1+x_{a}\right)(1-t)\right. \\
& \left.-\left(d_{\infty}+1\right)\left(1-x_{a}\right)(1+t)\right)\left(1+x_{a} t\right)-2 s_{a} x_{a}^{2}\left(1-t^{2}\right) .
\end{aligned}
$$

We remark that if $s_{b} \neq s_{a}, x_{b}$ cannot equal $x_{a}$, since $\int_{-1}^{1} p_{\mathrm{c}}(t)\left(1-t^{2}\right) d t$ is positive. Hence if $x_{a}=x_{b}$, then $s_{a}=s_{b}$ and $S_{a} \times S_{b}$ is Kähler-Einstein. Thus we do not need to check that $x_{a}$ are distinct: if $x_{a}=x_{b}$, we still get a WBF Kähler metric, but the hamiltonian 2-form has fewer constant roots.

Note also that we can replace the momentum coordinate $z$ by $-z$ : this allows us to replace $s_{a}$ by $-s_{a}$ and $x_{a}$ by $-x_{a}$, provided we interchange $d_{0}$ and $d_{\infty}$.

Remark 4.1. If the base manifolds are all $\mathbb{C} P^{d_{a}}$ and come in pairs with equal dimensions with $d_{0}=d_{\infty}$ and (say) $d_{2 k-1}=d_{2 k}$ for $k \geq 1$, then it is straightforward to find some Kähler-Einstein solutions to the equations $h_{a}=$ 0 by symmetry: for $\left|q_{a}\right|<\left(d_{a}+1\right) /(d+1)$ with $q_{2 j-1}=-q_{2 j}$, set $s_{a}=\left(d_{a}+\right.$ $1) / q_{a}$ and $x_{a}=q_{a}(d+1) /\left(d_{a}+1\right)$; then the integrand defining $h_{a}$ is an odd function of $t$, hence $h_{a}=0$. These metrics are special cases of those of KoisoSakane $[12,13]$ and provide examples where the necessary and sufficient conditions of Corollary 3.1 are verified (see also Corollary 4.2 below).

\subsection{WBF Kähler metrics over a Kähler-Einstein manifold}

Let us consider the case when the base is a single Kähler-Einstein manifold i.e., $\# \mathcal{A}=1$. In the absence of blow-downs, this case was also considered in [3]. Dropping the $a$ subscript for this unique $a \in \mathcal{A}$, we may assume that we have to find $0<x<1$ such that $h(x)=0$, where

$$
\begin{aligned}
h(x)= & \int_{-1}^{1}(1+t)^{d_{0}}(1-t)^{d_{\infty}}(1+x t)^{d} k(x, t) d t \\
k(x, t)= & \left(\left(d_{0}+1\right)(1+x)(1-t)-\left(d_{\infty}+1\right)(1-x)\right. \\
& \times(1+t))(1+x t)-2 s x^{2}\left(1-t^{2}\right) .
\end{aligned}
$$

(Alternatively we could assume that e.g., $d_{0} \leq d_{\infty}$, but then both $x$ positive and $x$ negative have to be considered.) Since $(1+t)^{d_{0}+1}(1-t)^{d_{\infty}+1}$ 
$(1+x t)^{d+1}(1-x t)$ vanishes at $t= \pm 1$ we may add its derivative onto the integrand to obtain

$$
\begin{aligned}
h(x) & =\int_{-1}^{1}(1+t)^{d_{0}+1}(1-t)^{d_{\infty}+1}(1+x t)^{d} x \hat{k}(x, t) d t \\
\hat{k}(x, t) & =\left(d_{0}+d_{\infty}+2-d\right)(1+x t)+2 x((d+1) t-s) .
\end{aligned}
$$

Using the two integral formulae for $h(x)$, we make the following observations:

- $h(1)$ has sign $\left(d_{0}+1\right)-s$;

- if $d \neq d_{0}+d_{\infty}+2, h(x)$ has sign $d_{0}+d_{\infty}+2-d$ for $x$ small and positive;

- if $d=d_{0}+d_{\infty}+2$, then $h(x)$ has $\operatorname{sign}(d+1)\left(d_{0}-d_{\infty}\right)-s(d+2)$ (if this is nonzero) for small nonzero $x$.

For this last case, evaluating $h(x) / x^{2}$ at $x=0$ gives $(s+(d+1)) I_{0}$ $+(s-(d+1)) I_{\infty}$ where $I_{0}$ and $I_{\infty}$ are integrals related by the identity $\left(d_{0}+2\right) I_{0}=\left(d_{\infty}+2\right) I_{\infty}$.

If $d=d_{0}+d_{\infty}+2$ and $(d+1)\left(d_{0}-d_{\infty}\right)=s(d+2)$, it is easy to see (integrating (4.10) by parts) that there are no solutions of $h(x)=0$ with $0<x<1$.

Since $h$ is continuous, these sign observations lead to existence results.

Theorem 4.1. Let $\left(S, g_{S}, \omega_{S}\right)$ be a compact Hodge Kähler-Einstein 2dmanifold of scalar curvature $2 d s$ and let $E_{0}, E_{\infty}$ be projectively flat hermitian vector bundles of ranks $d_{0}+1, d_{\infty}+1$ over $S$ with with $\bar{c}_{1}\left(E_{\infty}\right)-\bar{c}_{1}\left(E_{0}\right)=$ $\left[\omega_{S} / 2 \pi\right]$. Then there is an admissible WBF Kähler metric on $P\left(E_{0} \oplus E_{\infty}\right) \rightarrow$ $S$ when:

- $S$ has nonpositive scalar curvature $(s \leq 0), d \geq d_{0}+d_{\infty}+2$, unless $d=d_{0}+d_{\infty}+2$ and $(d+1)\left(d_{\infty}-d_{0}\right) \leq|s|(d+2)$

- $S$ has positive scalar curvature $(s>0),\left(d_{0}+1\right)>s$ and $d \geq d_{0}+$ $d_{\infty}+2$, unless $d=d_{0}+d_{\infty}+2$ and $d_{0}>d_{\infty} ;$

- $S$ has positive scalar curvature $(s>0),\left(d_{0}+1\right)<s$ and $d<d_{0}+$ $d_{\infty}+2$

When $d_{0}=d_{\infty}=0$ and $S$ is a positive Kähler-Einstein manifold, these existence results are sharp. In particular, when $S=\mathbb{C} P^{d}$, we obtain the following result. 
Theorem 4.2. There is a WBF Kähler metric on $P(\mathcal{O} \oplus \mathcal{O}(q)) \rightarrow \mathbb{C} P^{d}$ with $q>0$ if and only if $d=1$ and $q=1$ or $d \geq 2$ and $q>d+1$. The WBF Kähler metric is then unique up to automorphism and scale.

Proof. Any WBF Kähler metric is extremal and the extremal Kähler metrics on $M=P(\mathcal{O} \oplus \mathcal{O}(q)) \rightarrow \mathbb{C} P^{d}$ have cohomogeneity one under a maximal compact connected subgroup of $\operatorname{Aut}(M, J)[7]$. Since any two such subgroups are conjugate in the connected component $\operatorname{Aut}(M, J)^{0}$, it follows that, up to pullback by a automorphism, the WBF Kähler metrics on these manifolds must be admissible. The existence of a WBF Kähler metric in the stated cases follows from Theorem 4.1 above, so it remains to establish the nonexistence and uniqueness results.

For the case $d=1$, we compute that

$$
h(x)=\frac{4}{3} x\left(x^{2}+1-2 s x\right)
$$

and clearly there is a unique solution $0<x<1$ to $h(x)=0$ iff $s>1$. Since $S$ in this case is $\mathbb{C} P^{1}, \mathcal{K}^{-1}=\mathcal{O}(2)$ and the only possibility is $s=2, \mathcal{L}=\mathcal{O}(1)$, in accordance with the classification of [1].

For the case $d=2$ we calculate directly that

$$
h(x)=\frac{8}{15} x^{2}\left(6 x-s\left(x^{2}+5\right)\right)
$$

and clearly there is a unique solution $0<x<1$ to $h(x)=0$ iff $0<s<1$.

We now assume $d \geq 3$ and compute the integral (e.g., by substitution) to get:

$$
\begin{aligned}
& -\frac{1}{2}(d+1)(d+2)(d+3) x^{2} h(x)= \\
& \quad(1-x)^{d+2}(d+1+((d+1)(d+2)+2 s) x+((d+1)(d+3) \\
& \left.\quad+2(d+2) s) x^{2}\right)-(1+x)^{d+2}(d+1 \\
& \left.\quad-((d+1)(d+2)-2 s) x+((d+1)(d+3)-2(d+2) s) x^{2}\right) .
\end{aligned}
$$

If $x=(y-1) /(y+1)$ and $f(y)=-(d+1)(d+2)(d+3)(y+1)^{d+1}(y-1)$ $h(x) / 2^{d+4}$ then

$$
\begin{aligned}
f(y)= & (d+1)(s+1)-(d+2)(d+1+2 s) y+(d+3)(d+1+s) y^{2} \\
& +y^{d+2}(-(d+3)(d+1-s)+(d+2)(1+d-2 s) y \\
& \left.+(d+1)(s-1) y^{2}\right) .
\end{aligned}
$$


The zeros of $h(x)$ in $(0,1)$ correspond to the zeros of $f(y)$ in $(1, \infty)$. The latter problem is more amenable to calculus, since $f(1)=f^{\prime}(1)=f^{\prime \prime}(1)=0$ and $f^{\prime \prime \prime}(y)=(d+1)(d+2)(d+3) y^{d-1} P(y)$, where

$$
P(y)=-d(1+d-s)+(d+2)(d+1-2 s) y+(d+4)(s-1) y^{2} .
$$

Now $P(1)=d-2$, which is positive for $d>2$, while $P(0)$ is nonpositive since $s \leq d+1$. Hence $P(y)$ is positive in $(1, \infty)$ unless $s<1$, in which case it has a unique zero. If $P(y)$ is positive in $(1, \infty)$, then so is $f^{\prime \prime \prime}$, hence $f^{\prime \prime}$, $f^{\prime}$ and $f$, because we know that $f(1)=f^{\prime}(1)=f^{\prime \prime}(1)=0$. This gives the nonexistence. Similarly, when $f^{\prime \prime \prime}(y)$ has a unique zero in $(1, \infty)$, so does $f$, which gives the required uniqueness.

Note that the proof above in the case $d=2$ also gives us the following result.

Theorem 4.3. Let $S$ be a compact Kähler-Einstein complex surface. There is an admissible WBF Kähler metric with $\# \mathcal{A}=1$ on $P(\mathcal{O} \oplus \mathcal{L}) \rightarrow S$ if and only if $S$ is a positive Kähler-Einstein manifold and $\mathcal{L}=\mathcal{K}^{-q / p}$, where integers with $|q|>p>0$ such that $\mathcal{K}^{-1 / p}$ is the primitive ample root of the canonical bundle of $S$. The admissible WBF Kähler metrics is then unique up to automorphism and scale.

We end this paragraph by studying in more detail the case $d=1$ and $d_{0}+d_{\infty}=1$, when $M$ is a $\mathbb{C} P^{2}$-bundle over a compact Riemann surface $S_{1}=\Sigma$. Again, we assume without loss that $0<x<1$.

When $d_{0}=1$ and $d_{\infty}=0$ we have $h(x)=0$ iff $r(x)=(3-s) x^{2}+(4-$ $5 s) x+5=0$. If $r(x)=0$ then $s \geq 4 / 5$ and the (positive definite) metric $g_{\Sigma}$ is a constant curvature metric on $\Sigma=\mathbb{C} P^{1}$, so we must have that $E_{0}=$ $\mathcal{L}_{0} \otimes \mathbb{C}^{2}, E_{\infty}=\mathcal{L}_{\infty} \otimes \mathbb{C}$ for some line bundles $\mathcal{L}_{0}, \mathcal{L}_{\infty}$ and that $\omega_{\Sigma} / 2 \pi$ is integral. Thus $s=1$ or $s=2$ (since $s=2 / q$ for $q \in \mathbb{Z}^{+}$). However $r(x)$ does not have a root in $(0,1)$ in either case.

When $d_{0}=0$ and $d_{\infty}=1$, we have $h(x)=0$ iff $r(x)=(3+s) x^{2}-$ $(4+5 s) x+5=0$. Since $r(x)=(1-x)(5-4 x)>0$ for $s=1$ and $\frac{\partial}{\partial s} r(x)=$ $x(x-5)<0, r(x)$ has no roots in $(0,1)$ for $s \leq 1$. Then we may assume that $S_{1}=\mathbb{C} P^{1}$ and that $E_{0}=\mathcal{O} \otimes \mathbb{C}=\mathcal{O}$ and $E_{\infty}=\mathcal{L} \otimes \mathbb{C}^{2}$, where $\mathcal{L}$ is a holomorphic line bundle with $c_{1}(\mathcal{L})=\left[\omega_{\Sigma} / 2 \pi\right]$. By integrality, the only possibility with $s>1$ is $s=2$, for which we find a unique solution $x=(7-2 \sqrt{6}) / 5$ in $(0,1)$ (so $\Sigma=\mathbb{C} P^{1}$ and $\mathcal{L}=\mathcal{O}(1)$ ). Observe that $B \neq 0$, so the corresponding metric is not Kähler-Einstein. 
Theorem 4.4. Let $E_{0}, E_{\infty}$ be projectively flat hermitian vector bundles over a compact Riemann surface $\Sigma$ with ranks $d_{0}+1, d_{\infty}+1$, respectively. Then there is an admissible WBF Kähler metric on $P\left(E_{0} \oplus E_{\infty}\right) \rightarrow \Sigma$ with $d_{0}+d_{\infty}=1$ if and only if (without loss) $d_{0}=0, d_{\infty}=1, \Sigma=\mathbb{C} P^{1}$ and $E_{0}=\mathcal{O}$ while $E_{\infty}=\mathcal{O}(1) \otimes \mathbb{C}^{2}$. The admissible WBF Kähler metric is then unique up to automorphism and scale.

\subsection{WBF Kähler metrics over a product of Kähler-Einstein manifolds}

In this paragraph and the next, we consider the case that $d_{0}=d_{\infty}=0$ and $\# \mathcal{A}=2$ in detail. We will assume that the base $S$ is a global product of two Kähler Einstein manifolds $S_{a}(a=1,2)$ of dimensions $2 d_{a}>0$. We postpone a detailed discussion of the case $d_{1}=d_{2}=1$ to the next paragraph (where we also consider the case where $S$ is a local product). In this setting we have (up to a constant factor)

$$
\begin{aligned}
& h_{1}\left(x_{1}, x_{2}\right)=\int_{-1}^{1}\left(1+x_{1} z\right)^{d_{1}}\left(1+x_{2} z\right)^{d_{2}}\left(x_{1}\left(x_{1} s_{1}-1\right)\left(1-z^{2}\right)+z\left(1-x_{1}^{2}\right)\right) d z \\
& h_{2}\left(x_{1}, x_{2}\right)=\int_{-1}^{1}\left(1+x_{1} z\right)^{d_{1}}\left(1+x_{2} z\right)^{d_{2}}\left(x_{2}\left(x_{2} s_{2}-1\right)\left(1-z^{2}\right)+z\left(1-x_{2}^{2}\right)\right) d z .
\end{aligned}
$$

We are looking for common zeros of these functions with $0<\left|x_{a}\right|<1$. Let us note what we know about these functions on the boundary of this domain:

- when $x_{1}=0, h_{1}$ has the same sign as $x_{2}$;

- when $x_{1}= \pm 1, h_{1}$ has the same sign as $s_{1} \mp 1$;

- when $x_{2}=0, h_{2}$ has the same sign as $x_{1}$;

- when $x_{2}= \pm 1, h_{2}$ has the same sign as $s_{2} \mp 1$.

In particular, the curves $h_{1}=0$ and $h_{2}=0$ both pass through $(0,0)$ and we know the gradients of these curves at $(0,0)$, since $\partial h_{a} / \partial x_{a}=2\left(d_{a}-2\right) / 3$ and $\partial h_{a} / \partial x_{b}=2 d_{b} / 3$ for $b \neq a$. Hence along $h_{1}=0$ we have $d x_{2} / d x_{1}=(2-$ $\left.d_{1}\right) / d_{2} \leq 1$ at $(0,0)$, while along $h_{2}=0$ we have $d x_{1} / d x_{2}=\left(2-d_{2}\right) / d_{1} \leq 1$ at $(0,0)$ so that $d x_{2} / d x_{1}=d_{1} /\left(2-d_{2}\right)$ (infinite when $\left.d_{2}=2\right)$. Furthermore, if both curves have negative gradients, $d x_{2} / d x_{1}$, at $(0,0)$ - that is, both curves emanate from the origin into the fourth quadrant - then we must have that $d_{1}>2$ and $d_{2}>2$. Hence the difference in the gradients, namely $2\left(d_{1}+d_{2}-2\right) / d_{2}\left(d_{2}-2\right)$, is positive, so that the curve $h_{1}=0$ is above the curve $h_{2}=0$ for $x_{1}>0$ near $(0,0)$. 

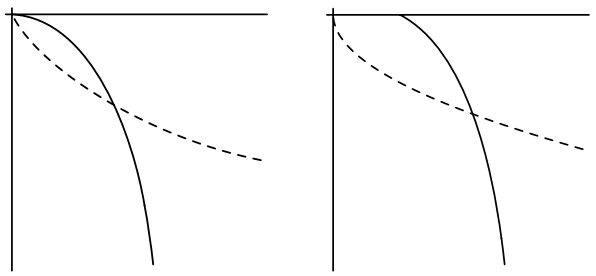

Figure 1: $d_{1}=2, d_{2}=3, s_{1}=3, s_{2}=-2$ and $d_{1}=1, d_{2}=2, s_{1}=2$, $s_{2}=-2$.

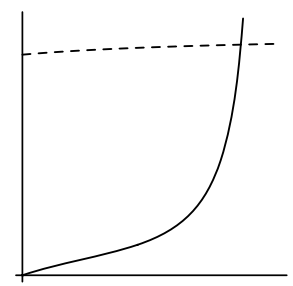

Figure 2: $d_{1}=1, d_{2}=3, s_{1}=2 / 3, s_{2}=4 / 5$.

There are two separate types of solutions to seek: those with $x_{1}$ and $x_{2}$ of opposite sign, and those with $x_{1}$ and $x_{2}$ of the same sign. Figures 1, 2 plot examples of the graphs of $h_{1}=0$ (solid) and $h_{2}=0$ (dashed) in each case.

We consider first the case of opposite signs, and without loss, we seek solutions with $x_{1}>0$ and $x_{2}<0$. Suppose now that $s_{1}>1$ and $s_{2}<-1$. Then

- $h_{1}$ changes sign on any path from $x_{1}=0, x_{2}<0$ to $x_{1}=1, x_{2} \leq 0$;

- $h_{2}$ changes sign on any path from $x_{2}=0, x_{1}>0$ to $x_{2}=-1, x_{1} \geq 0$.

It follows by continuity that the curves $h_{1}=0$ and $h_{2}=0$ must cross.

Lemma 4.1. If $s_{1}>1$ and $s_{2}<-1$ then there exist $x_{1} \in(0,1), x_{2} \in(-1,0)$ such that $h_{1}\left(x_{1}, x_{2}\right)=0=h_{2}\left(x_{1}, x_{2}\right)$.

Proof. Since $h_{1}$ is negative on the half-line $\left(x_{1}=0, x_{2}<0\right)$ and positive on $x_{1}=1$, there is a connected component $\mathcal{C}$ of the curve $h_{1}=0$ in the square $(0,1) \times[0,-1]$ which crosses $x_{2}=-1$ for some $x_{1} \in(0,1)$, and it either crosses $x_{2}=0$ for some $x_{1} \in(0,1)$, or it emanates from the origin, and, within the square, is initially above the curve $h_{2}=0$, as in figure 1 . 
It follows that $h_{2}$ changes sign on $\mathcal{C}$, hence vanishes by continuity and connectedness.

Let us turn now to the case that $x_{1}$ and $x_{2}$ have the same sign, so without loss, $x_{1}>0$ and $x_{2}>0$. Suppose that $s_{1}<1$ and $s_{2}<1$. Then

- $h_{1}$ changes sign on any path from $x_{1}=0, x_{2}>0$ to $x_{1}=1, x_{2} \geq 0$;

- $h_{2}$ changes sign on any path from $x_{2}=0, x_{1}>0$ to $x_{2}=1, x_{1} \geq 0$;

- the curve $h_{1}=0$ lies below the line $x_{1}=x_{2}$ for $x_{1}>0$ near $(0,0)$, and is strictly below unless $d_{1}=1$;

- the curve $h_{2}=0$ lies above the line $x_{1}=x_{2}$ for $x_{2}>0$ near $(0,0)$, and is strictly above unless $d_{2}=1$.

Again we see that the curves $h_{1}=0$ and $h_{2}=0$ must cross, except perhaps in the case $d_{1}=d_{2}=1$, which we shall consider in the next paragraph.

Lemma 4.2. If $s_{1}<1$ and $s_{2}<1$, and $d_{1}, d_{2}$ are not both 1 , then there exist $x_{1}, x_{2} \in(0,1)$ such that $h_{1}\left(x_{1}, x_{2}\right)=0=h_{2}\left(x_{1}, x_{2}\right)$.

Proof. As in the previous lemma, there is a connected component $\mathcal{C}$ of the curve $h_{1}=0$ in the square $(0,1) \times[0,1]$ which crosses $x_{2}=1$ for some $x_{1} \in$ $(0,1)$, and it either crosses $x_{2}=0$ for some $x_{1} \in(0,1)$, or it emanates from the origin. In the latter case, we need to know that $h_{1}=0$ is initially below $h_{2}=0$, so that $h_{2}$ is initially positive. Since not both $d_{1}$ and $d_{2}$ equal one, this follows from the observations prior to the statement of the lemma.

Let us summarize what we have established, excluding the case $d_{1}=$ $d_{2}=1$.

Theorem 4.5. Let $S_{a}(a=1,2)$ be compact Kähler-Einstein $2 d_{a}$-manifolds with $d_{a} \geq 1$ not both one. Let $\mathcal{K}_{a}$ be the canonical bundles, and suppose (without loss unless $S_{a}$ is Ricci-flat) that the Kähler form $\pm \omega_{a}$ is integral. Let $\mathcal{L}_{a}$ be line bundles on $S_{a}$ with $c_{1}\left(\mathcal{L}_{a}\right)=\left[\omega_{a} / 2 \pi\right]$ and, if $S_{a}$ is not Ricciflat, let $\mathcal{L}_{a}$ be $\mathcal{K}_{a}^{-q_{a} / p_{a}}$ tensored by a flat line bundle, for integers $p_{a}, q_{a}$ where $\mathcal{K}^{-1 / p_{a}}$ is the primitive ample root of the canonical bundle of $S_{a}$. Then there is an admissible WBF Kähler metric on $P\left(\mathcal{O} \oplus \mathcal{L}_{1} \otimes \mathcal{L}_{2}\right) \rightarrow S_{1} \times S_{2}$ in the following cases:

- $S_{1}$ and $S_{2}$ have positive scalar curvature, $0<q_{1}<p_{1}$ and $0<-q_{2}<p_{2}$;

- for $a=1,2, q_{a}>p_{a}$ if $S_{a}$ has positive scalar curvature, $q_{a}>0$ if $S_{a}$ has negative scalar curvature and $\omega_{a}$ is positive if $S_{a}$ is Ricci flat. 
Corollary 4.1. There is a WBF Kähler metric on $P\left(\mathcal{O} \oplus \mathcal{O}\left(q_{1}, q_{2}\right)\right) \rightarrow$ $\mathbb{C} P^{d_{1}} \times \mathbb{C} P^{d_{2}}$ in the following cases:

- $q_{1}>d_{1}+1$ and $q_{2}>d_{2}+1$;

- $1 \leq q_{1} \leq d_{1}$ and $1 \leq-q_{2} \leq d_{2}$.

We will see in the next paragraph that this corollary also holds for $d_{1}=$ $d_{2}=1$. We conjecture that all WBF Kähler metrics on $P\left(\mathcal{O} \oplus \mathcal{O}\left(k_{1}, k_{2}\right)\right) \rightarrow$ $\mathbb{C} P^{d_{1}} \times \mathbb{C} P^{d_{2}}$ are given by this corollary and that the metric is unique (up to automorphism and scale) in each case. As in Theorem 4.2, extremal Kähler metrics on these manifolds are cohomogeneity one, hence of linear type, but unless $d_{1}=d_{2}=1$ (see next paragraph) we have not been able to establish the relevant nonexistence and uniqueness results for solutions of $h_{1}=0=h_{2}$.

We note also that if $d_{1}=d_{2}$ (including the case $d_{1}=d_{2}=1$ ) and $k_{1}=$ $-k_{2}$ in the above corollary, we have not just a WBF Kähler metric, but a Kähler-Einstein metric, as found by Koiso and Sakane [12,13,15].

Corollary $4.2[12,13,15] . \quad$ On $P(\mathcal{O} \oplus \mathcal{O}(q,-q)) \rightarrow \mathbb{C} P^{d} \times \mathbb{C} P^{d}$, with $1 \leq$ $q \leq d$, there is a Kähler-Einstein metric, given (on a dense open set) by

$$
\begin{aligned}
g= & \left(\frac{d+1}{q}+z\right) g_{1}+\left(\frac{d+1}{q}-z\right) g_{2}+\frac{z^{2}-(d+1)^{2} / q^{2}}{F(z)} d z^{2} \\
& +\frac{F(z)}{z^{2}-(d+1)^{2} / q^{2}} \theta^{2},
\end{aligned}
$$

where $\left(g_{1}, \omega_{1}\right)$ and $\left(g_{2}, \omega_{2}\right)$ are Fubini-Study metrics on the $\mathbb{C} P^{d}$ factors with holomorphic sectional curvature $2 / q, d \theta=\omega_{1}-\omega_{2}$ and $F(z)=\int_{-1}^{z} 2 t$ $\left(\frac{(d+1)^{2}}{q^{2}}-t^{2}\right) d t=-\frac{(d+1)^{2}}{q^{2}}\left(1-z^{2}\right)+\frac{1}{2}\left(1-z^{4}\right)$.

Proof. Let $s_{1}=-s_{2}=\frac{d+1}{q}$ and $x_{1}=-x_{2}=\frac{q}{d+1}$. Then clearly $h_{1}\left(x_{1}, x_{2}\right)=$ $h_{2}\left(x_{1}, x_{2}\right)=0$. Further, $x_{a}=1 / s_{a}$ so the WBF metric is Kähler-Einstein. 


\subsection{WBF Kähler metrics over a ruled surface}

Let us now consider the case $d_{1}=d_{2}=1$, when the base is a product of Riemann surfaces. Thus we have

$$
\begin{aligned}
& h_{1}\left(x_{1}, x_{2}\right)=\int_{-1}^{1}\left(x_{1} z+1\right)\left(x_{2} z+1\right)\left(x_{1}\left(x_{1} s_{1}-1\right)\left(1-z^{2}\right)+z\left(1-x_{1}^{2}\right)\right) d z \\
& h_{2}\left(x_{1}, x_{2}\right)=\int_{-1}^{1}\left(x_{1} z+1\right)\left(x_{2} z+1\right)\left(x_{2}\left(x_{2} s_{2}-1\right)\left(1-z^{2}\right)+z\left(1-x_{2}^{2}\right)\right) d z
\end{aligned}
$$

(up to a constant factor), which by integration gives

$$
\begin{aligned}
& h_{1}\left(x_{1}, x_{2}\right)=\frac{2}{15}\left(5 x_{2}-5 x_{1}+10 s_{1} x_{1}^{2}-7 x_{1}^{2} x_{2}-5 x_{1}^{3}+2 s_{1} x_{1}^{3} x_{2}\right) \\
& h_{2}\left(x_{1}, x_{2}\right)=\frac{2}{15}\left(5 x_{1}-5 x_{2}+10 s_{2} x_{2}^{2}-7 x_{2}^{2} x_{1}-5 x_{2}^{3}+2 s_{2} x_{2}^{3} x_{1}\right) .
\end{aligned}
$$

Without loss, we look for solutions to $h_{1}\left(x_{1}, x_{2}\right)=0=h_{2}\left(x_{1}, x_{2}\right)$ with $x_{1}>x_{2}$ and $x_{1}>0$. Solving $h_{1}=0$ for $s_{1}$, we find that $s_{1}$ must be positive, hence $s_{1}=2 / q_{1}$ for some integer $q_{1} \geq 1$. We then establish the following three lemmas, the proofs of which can be found in Appendix 5.

Lemma 4.3. If $s_{1}=2$ then there exist $\left(x_{1}, x_{2}\right) \in(0,1) \times(-1,1)$ such that $h_{1}\left(x_{1}, x_{2}\right)=h_{2}\left(x_{1}, x_{2}\right)=0$ iff $s_{2} \leq-2$. Moreover, in this case the solution is unique. If $s_{2}<-2$ the solution is in $(0,1) \times(0,1)$, i.e., $x_{2}>0$, while if $s_{2}=-2$, the solution is $\left(\frac{1}{2},-\frac{1}{2}\right)$.

Lemma 4.4. If $s_{1}=1$ then there exist $\left(x_{1}, x_{2}\right) \in(0,1) \times(-1,1)$ such that $h_{1}\left(x_{1}, x_{2}\right)=h_{2}\left(x_{1}, x_{2}\right)=0$ iff $s_{2}<-1$. Moreover, in this case the solution is unique and $x_{2}>0$.

Lemma 4.5. If $s_{1}=2 / q_{1}$, where $q_{1} \in \mathbb{Z}$ and $q_{1} \geq 3$, then there exist $\left(x_{1}, x_{2}\right)$ $\in(0,1) \times(-1,1)$ such that $h_{1}\left(x_{1}, x_{2}\right)=h_{2}\left(x_{1}, x_{2}\right)=0$ (with $s_{2}=2 / q_{2}$ if $\left.x_{2}>0\right)$ iff $-s_{1}<s_{2}<1$. Moreover, in this case the solution is unique and $x_{2}>0$.

We do not need to assume $S_{1}$ and $S_{2}$ are compact for these arguments. However, if $S_{1}$ is complete, it must be $\mathbb{C} P^{1}$ and the product $S_{1} \times S_{2}$ is a (trivial) ruled surface. More generally we can suppose this is the universal cover of compact Kähler surface, which is then a geometrically ruled surface $S=P(E)$ over a Riemann surface $\Sigma$ with universal cover $S_{2}$. It is well known that the existence of a local product metric on $S$ is equivalent to $P(E) \rightarrow \Sigma$ admitting a flat projective unitary connection. This in turn, by a famous 
result of Narasimhan and Seshadri [14], is equivalent to polystability of $E$. The above lemmas therefore imply the following result.

Theorem 4.6. Let $S$ be a Hodge 4-manifold whose universal cover is a product of constant curvature Riemann surfaces and suppose that $M=$ $P(\mathcal{O} \oplus \mathcal{L}) \rightarrow S$ has an admissible WBF Kähler metric. Then $S$ is a geometrically ruled surface $P(E)$ such that $E \rightarrow \Sigma$ is polystable. Let $\mathbf{f}, \mathbf{v}=$ $c_{1}(V P(E)) \in H^{2}(S, \mathbb{Z})$ denote the classes of a fibre of $P(E) \rightarrow \Sigma$ and of the vertical line bundle. We then have $c_{1}(\mathcal{L})=\left(q_{1} / 2\right) \mathbf{v}+q_{2} \mathbf{f}$ where $q_{1} \in \mathbb{Z}$, and $q_{2} \in \mathbb{Z}$ unless $q_{1}$ is odd and $E \rightarrow \Sigma$ is not spin (which may only happen when $\Sigma$ has genus $\mathbf{g}>1)$, in which case $q_{2}+1 / 2 \in \mathbb{Z}$. Furthermore, up to replacing $\mathcal{L}$ by $\mathcal{L}^{-1}$ :

- if $\Sigma=\mathbb{C} P^{1}, S=\mathbb{C} P^{1} \times \mathbb{C} P^{1}$ and we either have $q_{1}=1$ and $q_{2}=-1$, or we have $q_{1}, q_{2}>2$;

- if $\Sigma=T^{2}, q_{1}>2$ and $q_{2}>0$;

- if $\Sigma$ has genus $\mathbf{g}>1$, we either have $q_{1}>2$ and $q_{2}>q_{1}(\mathbf{g}-1)$, or we have $q_{1} \in\{1,2\}$ and $0<q_{2}<q_{1}(\mathbf{g}-1)$.

Conversely, in each case there is a unique admissible WBF Kähler metric on $M$ up to automorphism and scale.

Note that $E$ spin means that $\operatorname{deg} E$ is even. Since $\operatorname{deg}(E \otimes L)=\operatorname{deg} E+$ $2 \operatorname{deg} L$, this condition (like polystability) is independent of the choice of $E$ with $S=P(E)$.

Proof. We have seen already that $S=P(E)$ for $E \rightarrow \Sigma$ polystable. If $\Sigma=$ $\mathbb{C} P^{1}, E$ is trivial and $S=\mathbb{C} P^{1} \times \mathbb{C} P^{1}$. If $\Sigma=T^{2}$, without loss $E$ is either $\mathcal{O} \oplus \mathcal{E} \rightarrow \Sigma$ with $\operatorname{deg} \mathcal{E}=0$ or the nontrivial extension of $\mathcal{O} \rightarrow \Sigma$ [16]. In either case $\operatorname{deg} E=0$. Thus the non-spin case may only happen when the genus of $\Sigma$ is at least 2 .

Let $\omega_{\mathbb{C} P^{1}}$ be the Kähler form of the Fubini-Study metric on $\mathbb{C} P^{1}$ with volume one and let $\omega_{\Sigma}$ be a Kähler form of a CSC Kähler metric on $\Sigma$ of volume one.

Let $\mathbb{C} P^{1} \times \tilde{\Sigma} \rightarrow S$ denote the universal cover of $S$ (so $\tilde{\Sigma}$ covers $\Sigma$ ) and let $\pi_{1}: \mathbb{C} P^{1} \times \tilde{\Sigma} \rightarrow \mathbb{C} P^{1}$ denote the projection to the first factor. Then $\pi_{1}^{*} \omega_{\mathbb{C} P^{1}}$ descends to a closed $(1,1)$-form on $S$ which represents $\mathbf{v} / 2$, whereas $\mathbf{f}=\left[\pi^{*} \omega_{\Sigma}\right]$. Hence we see that a local product $q_{1} \omega_{\mathbb{C} P^{1}}+q_{2} \omega_{\Sigma}$ corresponds to a line bundle $\mathcal{L} \rightarrow S$ with Chern class $\left(q_{1} / 2\right) \mathbf{v}+q_{2} \mathbf{f} \in H^{2}(S, \mathbb{Z})$. Now we note that $H^{2}(S, \mathbb{Z})=\mathbb{Z} \mathbf{h} \oplus \mathbb{Z} \mathbf{f}$, where $\mathbf{h} \in H^{2}(S, \mathbb{Z})$ denotes the class of the dual of the (E-dependent) tautological line bundle on $S$ (see e.g., [9]). Since 
$\mathbf{v}=2 \mathbf{h}+(\operatorname{deg} E) \mathbf{f}$, the integrality condition on $q_{1}, q_{2}$ for the existence of $\mathcal{L}$ follows immediately. Now we apply Lemmas $4.3-4.5$, bearing in mind that $s_{1}=2 / q_{1}$ and $s_{2}=2(1-\mathbf{g}) / q_{2}$.

Corollary 4.3. There is a WBF Kähler metric (unique up to automorphism and scale $)$ on $P\left(\mathcal{O} \oplus \mathcal{O}\left(q_{1}, q_{2}\right)\right) \rightarrow \mathbb{C} P^{1} \times \mathbb{C} P^{1}$ if and only if $q_{1}>2$ and $q_{2}>2$, or $q_{1}=1$ and $q_{2}=-1$, the latter metric being Kähler-Einstein.

Proof. A WBF Kähler metric is in particular extremal and since extremal Kähler metrics on these manifolds are cohomogeneity one, hence admissible (up to automorphism), cf. [7], this follows from the above theorem and Corollary 4.2 .

\subsection{WBF versus extremal Kähler metrics}

Any WBF Kähler metric is extremal, so our results provide examples of extremal Kähler metrics in admissible Kähler classes in the sense of [4]. By the results of [4], we then obtain $N$-dimensional families of such metrics near a WBF metric, where $N$ is the number of Kähler-Einstein factors in the base. (In fact we do not need the base metrics $g_{a}$ to be Kähler-Einstein to get an extremal Kähler metric: it suffices in the above calculations that they are CSC and Hodge.)

\section{Classification of WBF Kähler metrics on compact 6-manifolds}

Using the theory of $[3,4]$, the results of the previous section yield the following classification result for compact 6-manifolds admitting WBF Kähler metrics.

Theorem 5.1. Suppose that $(M, J, g, \omega)$ is a compact connected WBF Kähler 6-manifold of order $\ell$. Then $\ell \in\{0,1\}$.

(i) If $\ell=0$ then $(M, J, g, \omega)$ is a local product of Kähler-Einstein manifolds.

(ii) If $\ell=1$, then $(M, J)$ is biholomorphic to one of the following.

(a) $P(\mathcal{O} \otimes \mathcal{L}) \rightarrow S$ where $S$ is a positive Kähler-Einstein complex surface, $\mathcal{L}=\mathcal{K}^{-q / p}$ and $q>p>0$ are integers and $p$ is the Fano index of $S$. 
(b) $P(\mathcal{O} \oplus \mathcal{L}) \rightarrow S$ where $S=P(E) \rightarrow \Sigma$ is a geometrically ruled surface such that $E \rightarrow \Sigma$ is polystable and $\mathcal{L}$ is given by Theorem 4.6, excluding $P(\mathcal{O} \oplus \mathcal{O}(-1,1)) \rightarrow \mathbb{C} P^{1} \times \mathbb{C} P^{1}$ (which arises in the case $\ell=0$ as it admits a Kähler-Einstein metric).

(c) $P\left(\mathcal{O} \oplus \mathcal{O}(1) \otimes \mathbb{C}^{2}\right) \rightarrow \mathbb{C} P^{1} \quad($ a blow-down of $P(\mathcal{O} \oplus \mathcal{O}(1,-1)) \rightarrow$ $\left.\mathbb{C} P^{1} \times \mathbb{C} P^{1}\right)$.

On each manifold in $(a)-(c)$, there is a unique WBF Kähler metric, up to automorphism and scale (and it has order 1 ).

Proof. In [4, Thm. 11] we proved that a compact extremal Kähler 6-manifold admitting a hamiltonian 2-form of order 2 with the extremal vector field tangent to the $\mathbb{T}^{c}$-orbits is isometric to $\mathbb{C} P^{3}$ with a Fubini-Study metric. On the other hand, a compact Kähler 6-manifold with a hamiltonian 2-form of order 3 is biholomorphic to $\mathbb{C} P^{3}$ [3], and hence, if it is extremal, it is again isometric to a Fubini-Study metric. Thus there are no compact WBF Kähler 6-manifolds of order 2 or 3.

Part (i) is immediate and the existence and biholomorphic classification in part (ii) follow from Theorems 4.3, 4.4 and 4.6. It remains to prove the uniqueness claim in (ii). By Theorems 4.3, 4.4 and 4.6, and the wellknown uniqueness result of Bando-Mabuchi for Kähler-Einstein metrics [5], it suffices to prove that any WBF Kähler metric is admissible (with the given bundle structures) up to scale and automorphism, for which, using [3] again, it is enough to show that the metric can be pulled back by an automorphism of $(M, J)$ so that the extremal vector field $J \operatorname{grad}_{g} \mathrm{Scal}_{g}$ becomes a nonzero multiple of the generator of the canonical $S^{1}$-action. We now establish the uniqueness in each case.

(a) By the classification of [17], $S$ is biholomorphic to $\mathbb{C} P^{2}, \mathbb{C} P^{1} \times \mathbb{C} P^{1}$ or a blow-up of $\mathbb{C} P^{2}$ at $k$ points in general position for $3 \leq k \leq 8$. When $S=\mathbb{C} P^{2}$ or $S=\mathbb{C} P^{1} \times \mathbb{C} P^{1}$, the uniqueness follows from Theorem 4.2 and Corollary 4.3, so it remains to consider the case that $S$ is a blow-up of $\mathbb{C} P^{2}$. This has Fano index $p=1$, so $\mathcal{L}=\mathcal{K}^{-q}$ for $q>1$. By Riemann-Roch, $H^{0}(S, \mathcal{L}) \neq 0$ while $H^{0}\left(S, \mathcal{L}^{-1}\right)=0$ since $\mathcal{L}$ is not trivial. Therefore, $[4$, Props. 3-4] show that $M$ does not admit any CSC Kähler metrics. In particular, any other WBF Kähler metric $g^{\prime}$ on $M$ must have order 1 and is therefore [3] admissible with respect to some ruling of $M$ over a KählerEinstein surface $S^{\prime}$ with $b_{2}\left(S^{\prime}\right)=b_{2}(M)-1=b_{2}(S)$. Since $g$ and $g^{\prime}$ are both extremal, by [7] we can assume, after pulling back $g^{\prime}$ by an automorphism, that $\mathfrak{i}_{0}\left(M, g^{\prime}\right)=\mathfrak{i}_{0}(M, g)$ in $\mathfrak{h}_{0}(M)$. Let $K, K^{\prime}$ be the extremal vector fields of $g, g^{\prime}$. Then $\mathcal{L}_{K} \mathrm{Scal}_{g^{\prime}}=\mathcal{L}_{K^{\prime}} \mathrm{Scal}_{g}=0$, so $K$ and $K^{\prime}$ commute and induce 
hamiltonian Killing vector fields $X, X^{\prime}$ on $S, S^{\prime}$. If either is zero, $K^{\prime}$ is a multiple of $K$ and we are done. Otherwise, $\mathfrak{h}(S), \mathfrak{h}\left(S^{\prime}\right) \neq 0$ so $S, S^{\prime}$ are both (isomorphic to) the blow-up of $\mathbb{C} P^{2}$ at three points. The corresponding Kähler-Einstein metrics agree up to automorphism and scale by [5], hence so do $g$ and $g^{\prime}$ (by Theorem 4.3).

(b)-(c) Here any Kähler class on $M$ is admissible, so $M$ admits no CSC Kähler metrics by [4, Thm. 5, Thm. 8 and Rem. 8] (in case (b), $\mathcal{L}$ is, without loss, ample by Theorem 4.6). Thus any WBF metric on $M$ has order 1. Being in an admissible class, its extremal vector field must be a multiple of $K$ by [4, Prop. 6].

Remark 5.1. In the classification of WBF Kähler 4-manifolds obtained in [1] the normalized Ricci form also has order 0 or 1. A naive dimension counting argument [3] supports the conjecture that this feature persists in higher dimensions. We also note that the base manifolds $S$ have Kodaira dimension $-\infty$. In view of the examples of Theorem 4.1 , this is no longer true in dimension $\geq 8$.

\section{Appendix. Proofs of Lemmas 4.3, 4.4 and 4.5}

This appendix gives the proofs of Lemmas 4.3, 4.4 and 4.5. The work here is basically a calculus marathon: while the existence of solutions in the stated cases is relatively straightforward, the nonexistence and uniqueness results are much more subtle.

We are looking for common zeros of the functions

$$
\begin{aligned}
& h_{1}\left(x_{1}, x_{2}\right)=\frac{2}{15}\left(5 x_{2}-5 x_{1}+10 s_{1} x_{1}^{2}-7 x_{1}^{2} x_{2}-5 x_{1}^{3}+2 s_{1} x_{1}^{3} x_{2}\right) \\
& h_{2}\left(x_{1}, x_{2}\right)=\frac{2}{15}\left(5 x_{1}-5 x_{2}+10 s_{2} x_{2}^{2}-7 x_{2}^{2} x_{1}-5 x_{2}^{3}+2 s_{2} x_{2}^{3} x_{1}\right) .
\end{aligned}
$$

with $0<x_{1}<1$ and $0<\left|x_{2}\right|<1$ (where $x_{2}$ is negative if $g_{2}$ is negative definite and positive if $g_{2}$ is positive definite). Since the equations $h_{1}, h_{2}=0$ are both of the form $y\left(5-7 x^{2}+2 s x^{3}\right)-5 x+10 s x^{2}-5 x^{3}=0$ we need to analyse the graphs of the functions $y=f_{s}(x):=\frac{5 x\left(x^{2}-2 s x+1\right)}{2 s x^{3}-7 x^{2}+5}$ for $-1<x<1$. Since also $\left|s_{a}\right|=2\left|\mathbf{g}_{a}-1\right| / q_{a}$, where $\mathbf{g}_{a}$ is the genus of the corresponding curve and $q_{a} \in \mathbb{Z}^{+}$, if $x_{a}$ is positive and $s_{a}>2 / 3$ then $s_{a} \in\{1,2\}$. Thus for $s>2 / 3$ we can restrict out attention to the case where $-1<x<0$ or $s \in\{1,2\}$. We then have the following lemma. 
Lemma 1. Let $\mathcal{C}=\mathcal{C}(s)$ denote the part of the graph of $y=f_{s}(x)=$ $\frac{5 x\left(x^{2}-2 s x+1\right)}{2 s x^{3}-7 x^{2}+5}$ which lies within the square $[-1,1] \times[-1,1]$. Then the following hold.

- When $0 \leq s \leq 2 / 3, \mathcal{C}$ looks like

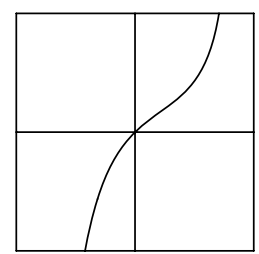

where the graph is convex for $x<0$, increasing everywhere, intersects the line $y=-1$ for some $-1<x<0$ and intersects $y=1$ for some $0<x<1$.

- When $s=1, \mathcal{C}$ looks like

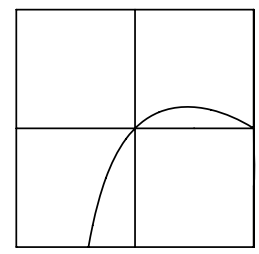

where the graph is convex everywhere, increasing for $x<0$, intersects the $x$-axis at $x=0$ and $x=1$ and intersects $y=-1$ for some $-1<$ $x<0$.

- When $s=2, \mathcal{C}$ looks like

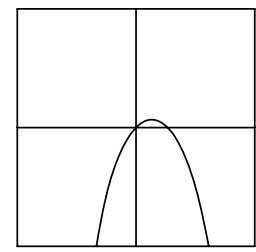

where the graph is convex everywhere, increasing for $x<0$, intersects the $x$-axis at $x=0$ and $x=2-\sqrt{3}$ and intersects $y=-1$ at $x=-1 / 3$ and $x=(5-\sqrt{10}) / 3$. 
- When $s \in(2 / 3,+\infty), \mathcal{C}$ restricted to $-1<x<0$ looks like

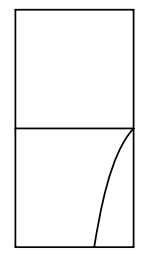

where the graph is convex and increasing, and intersects $y=-1$ for some $-1<x<0$.

Since $-f_{-s}(-x)=f_{s}(x)$, for $s<0, \mathcal{C}(s)$ is obtained by rotating $\mathcal{C}(-s)$ by $\pi$.

Proof. The cases $s=0, s=1$ and $s=2$ are elementary and will be omitted.

We first consider the graphs for $-1<x<0$. The numerator of $\frac{5 x\left(x^{2}-2 s x+1\right)}{2 s x^{3}-7 x^{2}+5}$ is strictly negative for $-1<x<0$, whereas the denominator is negative at $x=-1$, positive at $x=0$ and strictly increasing for $-1<$ $x<0$. We conclude that $f_{s}$ has precisely one asymptote $-1<a<0$ and $\lim _{x \rightarrow a^{ \pm}} f_{s}(x)=\mp \infty$. Also

$$
f_{s}^{\prime}(x)=\frac{5\left(5-20 s x+22 x^{2}-4 s x^{3}-7 x^{4}+4 s^{2} x^{4}\right)}{\left(5-7 x^{2}+2 s x^{3}\right)^{2}}
$$

is positive for $-1<x<0$ and since $f_{s}(-1)=5>1$ the graph of $f_{s}$ is outside the square $[-1,1] \times[-1,1]$ for $-1<x<a$. Thus we may restrict our attention to $a<x<0$ (and $f_{s}(x)$ is negative in this range). Now

$$
f_{s}^{\prime \prime}(x)=\frac{-20\left(25 s-90 x+135 s x^{2}-42 x^{3}-70 s^{2} x^{3}+51 s x^{4}-6 s^{2} x^{5}-7 s x^{6}+4 s^{3} x^{6}\right)}{\left(5-7 x^{2}+2 s x^{3}\right)^{3}}
$$

is negative for $a<x<0$ so $f_{s}$ is convex for $a<x<0$. Since $\lim _{x \rightarrow a^{+}} f_{s}(x)=$ $-\infty$ and $f_{s}(0)=0, \mathcal{C}$ must intersect the line $y=-1$ for some $-1<a<$ $x<0$.

It remains to consider $0<x<1$ and $0<s \leq 2 / 3$.

The denominator of $\frac{5 x\left(x^{2}-2 s x+1\right)}{2 s x^{3}-7 x^{2}+5}$ is a third degree polynomial which is negative at $x=-1$, positive at $x=0$, negative at $x=1$ and positive for $x \rightarrow$ $+\infty$, while the numerator is positive for $0<x<1$. We conclude that $f_{s}$ has precisely one asymptote $0<b<1, \lim _{x \rightarrow b^{ \pm}} f_{s}(x)=\mp \infty, f_{s}(x)>0$ for $0<$ $x<b$ and $f_{s}(x)<0$ for $b<x<1$. For $x \in[0,1] \backslash\{b\}$ the denominator of

$$
f_{s}^{\prime}(x)=\frac{5\left(5-20 s x+22 x^{2}-4 s x^{3}-7 x^{4}+4 s^{2} x^{4}\right)}{\left(5-7 x^{2}+2 s x^{3}\right)^{2}}
$$


is positive. For a fixed $0<x<1$, the numerator may be viewed as a function of $s$ and its derivative, $5 x\left(8 s x^{3}-4 x^{2}-20\right)$, with respect to $s$ is clearly negative. Since the value of the numerator of $f_{s}^{\prime}(x)$ at $s=2 / 3$ equals

$$
\begin{aligned}
\frac{5}{9}\left(45-120 x+198 x^{2}-24 x^{3}-47 x^{4}\right) & \\
= & \frac{5}{9}\left(47\left(x^{2}-x^{4}\right)+24\left(x^{2}-x^{3}\right)+\left(127 x^{2}-120 x+45\right)\right),
\end{aligned}
$$

which is positive, we conclude that if $0<s \leq 2 / 3, f_{s}^{\prime}(x)$ is positive for $x \in$ $[0,1] \backslash\{b\}$. Thus $f_{s}$ is strictly increasing. Since $f_{s}(1)=-5$ the graph of $f_{s}$ is outside the square $[-1,1] \times[-1,1]$ for $b<x<1$. Moreover, since $f_{s}(0)=$ 0 and $\lim _{x \rightarrow b^{-}} f_{s}(x)=+\infty, \mathcal{C}$ intersects the line $y=1$ for some $0<x<$ $b<1$.

It is clear from the shape of the graphs $\mathcal{C}(s)$ (corresponding to $h_{1}=0$ ) and their reflections in the line $y=x$ (corresponding to $h_{2}=0$ ) that the zero-sets of $h_{1}$ and $h_{2}$ intersect in the fourth quadrant $0<x_{1}<1,-1<$ $x_{2}<0$ iff $s_{1}=2$ and $s_{2}=-2$, and in this case they meet at a unique point $x_{1}=1 / 2, x_{2}=-1 / 2$. Hence we may assume from now on that $0<x_{2}<1$ and $s_{2} \leq 2$.

Let us now recall what we know about the functions $h_{1}$ and $h_{2}$ :

- the curves $h_{1}=0$ and $h_{2}=0$ both pass through $(0,0)$;

- along $h_{1}=0$ and $h_{2}=0$ we have $d x_{2} / d x_{1}=1$ at $(0,0)$;

- along $h_{1}=0$ we have $d^{2} x_{2} / d x_{1}^{2}=-4 s_{1}$ at $(0,0)$;

- along $h_{2}=0$ we have $d^{2} x_{2} / d x_{1}^{2}=4 s_{2}$ at $(0,0)$.

Therefore if $s_{2}>-s_{1}$ the zero-set of $h_{2}$ is above the zero-set of $h_{1}$ for $x_{1}$ small and positive, while if $s_{2}<-s_{1}$ it is below the zero-set of $h_{1}$ for $x_{1}$ small and positive.

By Lemma 1 , the zero-sets of $h_{2}$ in $(0,1) \times(0,1)$ look like
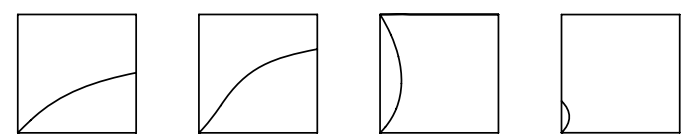

for $s_{2} \leq 0,0<s_{2} \leq 2 / 3, s_{2}=1$ and $s_{2}=2$, respectively. For $s_{2} \leq 2 / 3$ the zero-set of $h_{2}$ is an increasing graph which meets $x_{1}=1$ at a point with $0<x_{2}<1$.

It now follows easily that the zero-sets of $h_{1}$ and $h_{2}$ meet in at least one point $\left(x_{1}, x_{2}\right) \in(0,1) \times(0,1)$ in the following cases: 
- $s_{1} \in\{1,2\}, s_{2}<-s_{1}$;

- $0<s_{1} \leq 2 / 3,-s_{1}<s_{2} \leq 2 / 3$.

For the nonexistence and uniqueness results, assume that we do have a solution $\left(x_{1}, x_{2}\right) \in(0,1) \times(0,1)$ for $h_{1}=h_{2}=0$. Then from $h_{1}=0$, we have that

$$
x_{2}=\frac{5 x_{1}\left(x_{1}^{2}-2 s_{1} x_{1}+1\right)}{2 s_{1} x_{1}^{3}-7 x_{1}^{2}+5} .
$$

(It is easy to check that if $2 s_{1} x_{1}^{3}-7 x_{1}^{2}+5=0$ then we cannot have $h_{1}=0$ for $x_{1} \in(0,1)$.) If we substitute into $h_{2}=0$ we get

$$
\frac{10 x_{1}^{2}\left(x_{1}^{2}-5\right) \mathcal{M}\left(x_{1}, 1-x_{1}, s_{1}, s_{2}\right)}{\left(2 s_{1} x_{1}^{3}-7 x_{1}^{2}+5\right)^{3}}=0
$$

with

$$
\begin{aligned}
\mathcal{M}\left(x, y, s_{1}, s_{2}\right)= & -25 s_{1} y^{6}+30\left(2-5 s_{1}\right) x y^{5}+20\left(15-23 s_{1}\right) x^{2} y^{4} \\
& +8\left(72-105 s_{1}+25 s_{1}^{2}\right) x^{3} y^{3} \\
& +4\left(1-s_{1}\right)\left(132-125 s_{1}+25 s_{1}^{2}\right) x^{4} y^{2} \\
& +8\left(1-s_{1}\right)^{2}\left(36-25 s_{1}\right) x^{5} y+96\left(1-s_{1}\right)^{3} x^{6} \\
& -25 s_{2} y(2 x+y)\left(y^{2}+2\left(1-s_{1}\right) x(x+y)\right)^{2}
\end{aligned}
$$

Thus if $\left(x_{1}, x_{2}\right) \in(0,1) \times(0,1)$ is any solution of $h_{1}=h_{2}=0$, then $x_{1}$ must be a root of $\mathcal{M}\left(x_{1}, 1-x_{1}, s_{1}, s_{2}\right)$ and this determines $x_{2}$ uniquely. In the following $\mathcal{M}_{x}$ denotes the difference between the $x$ and $y$ derivatives of $\mathcal{M}$, so $\mathcal{M}_{x}\left(x, 1-x, s_{1}, s_{2}\right)$ is the $x$ derivative of $\mathcal{M}\left(x, 1-x, s_{1}, s_{2}\right) ; \mathcal{M}_{x x}$ is defined similarly.

Proof of Lemma 4.3. In this case $s_{1}=2$. We have seen that the zero-sets of $h_{1}$ and $h_{2}$ meet in $(0,1) \times(-1,0)$ iff $s_{2}=-2$ and then the intersection point is unique, being $(1 / 2,-1 / 2)$. We now analyse the case $x_{2}>0$. Any intersection point $\left(x_{1}, x_{2}\right) \in(0,1) \times(0,1)$ of the zero-sets of $h_{1}$ and $h_{2}$ must have $0<x_{1}<2-\sqrt{3}$ by Lemma .1, and $x=x_{1}$ must be a root of $\mathcal{M}(x, 1-$ $\left.x, 2, s_{2}\right)$ where

$$
\begin{aligned}
\mathcal{M}\left(x, y, 2, s_{2}\right)= & -96 x^{6}-112 x^{5} y+72 x^{4} y^{2}-304 x^{3} y^{3}-620 x^{2} y^{4}-240 x y^{5} \\
& -50 y^{6}-25 s_{2} y(2 x+y)\left(y^{2}-2 x(x+y)\right)^{2}
\end{aligned}
$$


Clearly $\mathcal{M}\left(x, 1-x, 2, s_{2}\right)$ is a decreasing function of $s_{2}$ when $0<x<2-$ $\sqrt{3}$. Since

$\mathcal{M}(x, z+x, 2,-2)=-4 x z\left(765 x^{4}+2040 x^{3} z+1846 x^{2} z^{2}+680 x z^{3}+85 z^{4}\right)$,

$\mathcal{M}(x, 1-x, 2,-2)<0$ for $0<x<2-\sqrt{3}<1 / 2$, hence so is $\mathcal{M}(x, 1-x$, $\left.2, s_{2}\right)$ for $s_{2} \geq-2$. Thus there are no solutions to $h_{1}=h_{2}=0$ in $(0,1) \times$ $(0,1)$ for $s_{2} \geq-2$.

Now suppose $s_{2}<-2$. We have seen that the zero-sets intersect in at least one point $\left(x_{1}, x_{2}\right) \in(0,1) \times(0,1)$. We now compute

$$
\begin{aligned}
\frac{\partial \mathcal{M}_{x x}}{\partial s_{2}}\left(x, z+x, 2, s_{2}\right) & =-25\left(9 x^{4}+216 x^{3} z+306 x^{2} z^{2}+136 x z^{3}+17 z^{4}\right) \\
\mathcal{M}_{x x}(x, z+2 x, 2,-2) & =8\left(4554 x^{4}+9340 x^{3} z+5757 x^{2} z^{2}+1311 x z^{3}+85 z^{4}\right),
\end{aligned}
$$

so $\mathcal{M}_{x x}\left(x, 1-x, 2, s_{2}\right)$ is a decreasing function of $s_{2}$ for $0<x<2-\sqrt{3}<$ $1 / 2$ whose value at $s_{2}=-2$ is positive for $0<x<2-\sqrt{3}<1 / 3$. Hence $\mathcal{M}\left(x, 1-x, 2, s_{2}\right)$ is a concave function of $x \in(0,2-\sqrt{3})$. At $x=0$ it equals $-25\left(2+s_{2}\right)>0$, while at $x=2-\sqrt{3}$ it equals $48(240-139 \sqrt{3})<0$. Hence it has exactly one root $x=x_{1} \in(0,2-\sqrt{3})$ and the solution to $h_{1}=h_{2}=0$ is unique.

Proof of Lemma 4.4. In this case $s_{1}=1$. We have seen that the zero-sets of $h_{1}$ and $h_{2}$ do not meet in $(0,1) \times(-1,0)$, so we restrict attention to $x_{2}>0$. Since

$$
\mathcal{M}\left(x, y, 1, s_{2}\right)=-y^{3}\left(64 x^{3}+160 x^{2} y+10\left(9+5 s_{2}\right) x y^{2}+25\left(1+s_{2}\right) y^{3}\right),
$$

there are no roots of $\mathcal{M}\left(x, 1-x, 1, s_{2}\right)$ in $(0,1)$ for $s_{2} \geq-1$. Suppose now that $s_{2}<-1$. We have seen that the zero-sets intersect in at least one point in $(0,1) \times(0,1)$. The difference between the $x$ and $y$ derivatives of $-\mathcal{M}\left(x, y, 1, s_{2}\right) / y^{3}$ is

$$
32 x^{2}+140 x y+15 y^{2}-25 s_{2} y(4 x+y),
$$

which is clearly positive for $x \in(0,1), y=1-x$ since $s_{2}$ is negative. Hence $-\mathcal{M}\left(x, 1-x, 1, s_{2}\right) /(1-x)^{3}$ is an increasing function of $x \in(0,1)$ so it has at most one root and the solution to $h_{1}=h_{2}=0$ is unique.

Proof of Lemma 4.5. In this case $s_{1}=2 / q_{1}, q_{1}=3,4,5, \cdots$. We have seen that the zero-sets of $h_{1}$ and $h_{2}$ do not meet in $(0,1) \times(-1,0)$. Thus we may 
assume $0<x_{2}<1$ and $s_{2} \leq 2 / 3$ : by the previous two lemmas (with $s_{1}, s_{2}$ interchanged) there are no solutions with $s_{2} \in\{1,2\}$.

If there were a solution $\left(x_{1}, x_{2}\right) \in(0,1) \times(0,1)$ to $h_{1}=h_{2}=0$ it would give a root $x=x_{1}$ of the function $\mathcal{M}\left(x, 1-x, s_{1}, s_{2}\right)$. We now observe that $\partial \mathcal{M} / \partial s_{2}$ is negative for $y=1-x, x \in(0,1)$ (since $s_{1}<1$ ) and that

$$
\begin{aligned}
\mathcal{M}(x, y, s,-s)= & 4 x((1-s) x+y) \mathcal{M}_{0}(x, y, s) \\
\mathcal{M}_{0}(x, y, s)=24 & (1-s)^{2} x^{4}+48(1-s) x^{3} y \\
& \quad+4\left(21-25 s^{2}\right) x^{2} y^{2}+20\left(3-5 s^{2}\right) x y^{3}+5\left(3-5 s^{2}\right) y^{4}
\end{aligned}
$$

so $\mathcal{M}(x, 1-x, s,-s)$ is positive on $(0,1)$ for $s \leq 2 / 3<\sqrt{3 / 5}$. Thus $\mathcal{M}(x, 1-$ $\left.x, s_{1}, s_{2}\right)$ is positive for $0<x<1$ and $s_{2} \leq-s_{1}$ and there are no solutions $\left(x_{1}, x_{2}\right)$ to $h_{1}=h_{2}=0$ with $0<x_{1}<1$ when $s_{2} \leq-s_{1}$.

We now let $s_{2}>-s_{1}$. We have seen that the zero-sets intersect in at least one point in $(0,1) \times(0,1)$. We want to show that they intersect in at most one point. The proof, which is harder than previously, is motivated by the observation that

$$
\begin{aligned}
\mathcal{M}(x, y, 2 / 3,2 / 3)= & \frac{4}{27}\left(3 x^{2}-x y-5 y^{2}\right) \\
& \times\left(8 x^{4}+8 x^{3} y+112 x^{2} y^{2}+120 x y^{3}+45 y^{4}\right)
\end{aligned}
$$

and hence $\mathcal{M}(x, 1-x, 2 / 3,2 / 3)$ is positive for $x_{0}<x<1$ where $x_{0}=(9-$ $\sqrt{61}) / 2$ is the smallest root of $x^{2}-9 x+5=0$. Observe that $x_{0} \approx 0.595$ is less than $3 / 5$ (since $1521=39^{2}$ is less than $1525=5^{2} \cdot 61$ ). We are going to prove that $\mathcal{M}\left(x, 1-x, s_{1}, s_{2}\right)>0$ for $3 / 5 \leq x<1$ and that $\mathcal{M}_{x}(x, 1-$ $\left.x, s_{1}, s_{2}\right)>0$ for $0<x \leq 3 / 5$. This will prove that there is at most one root on $(0,1)$.

Since $\mathcal{M}\left(x, 1-x, s_{1}, s_{2}\right)$ is a decreasing function of $s_{2}$, to prove positivity for $3 / 5 \leq x<1$, it suffices to prove $\mathcal{M}\left(x, 1-x, s_{1}, 2 / 3\right)>0$ for $3 / 5 \leq x<1$. This is true for $s_{1}=2 / 3$ and so the positivity follows from:

Claim 1. $\frac{\partial \mathcal{M}}{\partial s_{1}}\left(x, 1-x, s_{1}, 2 / 3\right)<0$ for $3 / 5 \leq x<1$.

The positivity of $\mathcal{M}_{x}\left(x, 1-x, s_{1}, s_{2}\right)$ on $0<x \leq 3 / 5$ for $-s_{1}<s_{2} \leq 2 / 3$ follows from the fact that it is an affine linear function of $s_{2}$ such that:

Claim 2. $\mathcal{M}_{x}\left(x, 1-x, s_{1}, 2 / 3\right)>0$ for $0<x \leq 3 / 5$;

Claim 3. $\mathcal{M}_{x}\left(x, 1-x, s_{1},-s_{1}\right)>0$ for $0<x \leq 3 / 5$.

Subject to these three claims, we are done. 
Proof of Claim 1. We compute that $-12 \frac{\partial \mathcal{M}}{\partial s_{1}}(z / 2+3 y / 2, y, s, 2 / 3)$ is given by

$$
\begin{aligned}
& 18\left(6665-11290 s+6237 s^{2}\right) y^{6}+\left(195155-361344 s+194157 s^{2}\right) y^{5} z \\
& +2\left(67267-131470 s+69255 s^{2}\right) y^{4} z^{2}+2\left(24931-50180 s+26055 s^{2}\right) y^{3} z^{3} \\
& +2\left(5213-10610 s+5445 s^{2}\right) y^{2} z^{4}+(1-s)(1163-1197 s) y z^{5}+54(1-s)^{2} z^{6} .
\end{aligned}
$$

It suffices to show that the coefficient of each monomial in $y, z$ is positive for $0<s \leq 2 / 3$ (put $y=1-x, z=5 x-3$ ). The first five quadratics in $s$ have no real roots and are positive at $s=0$, and for the last two the result is clear.

Proof of Claim 2. We compute that $\frac{729}{2} \mathcal{M}_{x}(x, z / 3+2 x / 3, s, 2 / 3)$ is given by

$$
\begin{gathered}
4\left(88050-255955 s+293700 s^{2}-99063 s^{3}\right) x^{5}+80\left(2460-4277 s+5862 s^{2}\right. \\
\left.-2025 s^{3}\right) x^{4} z+4\left(19530-3229 s+10575 s^{2}-4050 s^{3}\right) x^{3} z^{2}+4(4632 \\
\left.+2425 s-975 s^{2}\right) x^{2} z^{3}+5\left(420+347 s-120 s^{2}\right) x z^{4}+10(9+10 s) z^{5} .
\end{gathered}
$$

It suffices to show that the coefficient of each monomial in $x, z$ is positive for $0<s \leq 2 / 3$ (put $z=3-5 x$ ). For the two quadratics and the last coefficient, this is clear. The remaining three cubics are positive multiples of

$$
\begin{gathered}
44025(2-3 s)^{3}+140270(2-3 s)^{2} s+240345(2-3 s) s^{2}+251028 s^{3} \\
1230(2-3 s)^{3}+6793(2-3 s)^{2} s+19272(2-3 s) s^{2}+21789 s^{3} \\
9765(2-3 s)^{3}+84656(2-3 s)^{2} s+265431(2-3 s) s^{2}+281844 s^{3}
\end{gathered}
$$

Hence they are all positive on $[0,2 / 3]$.

Proof of Claim 3. We compute that $\frac{729}{4} \mathcal{M}_{x}(x, z / 3+2 x / 3, s,-s)$ is given by

$$
\begin{aligned}
& 48\left(7125-23940 s+23225 s^{2}-4974 s^{3}\right) x^{5}+240\left(1020-2259 s+1054 s^{2}\right. \\
& \left.\quad+525 s^{3}\right) x^{4} z+24\left(4080-4509 s-2750 s^{2}+4275 s^{3}\right) x^{3} z^{2}+24(897-450 s \\
& \left.\quad-1225 s^{2}+750 s^{3}\right) x^{2} z^{3}+30(25-6 s)\left(3-5 s^{2}\right) x z^{4}+30\left(3-5 s^{2}\right) z^{5} .
\end{aligned}
$$

It suffices to show that the coefficient of each monomial in $x, z$ is positive for $0<s \leq 2 / 3$. This is clear for the last two coefficients. The remaining 
four cubics are positive multiples of

$$
\begin{gathered}
7125(2-3 s)^{3}+16245(2-3 s)^{2} s-2005(2-3 s) s^{2}+363 s^{3} \\
510(2-3 s)^{3}+2331(2-3 s)^{2} s+2324(2-3 s) s^{2}+1863 s^{3} \\
2040(2-3 s)^{3}+13851(2-3 s)^{2} s+22526(2-3 s) s^{2}+15099 s^{3} \\
897(2-3 s)^{3}+7173(2-3 s)^{2} s+13919(2-3 s) s^{2}+7419 s^{3} .
\end{gathered}
$$

Only the first is not manifestly positive on $[0,2 / 3]$. However it is positive at the endpoints and (dividing by 8 ) the cubic $7125-23940 s+23225 s^{2}-$ $4974 s^{3}$ has a minimum at $s=(23225-\sqrt{182167945}) / 14922 \approx 0.652$ where it takes the value $5(492445959775-36433589 \sqrt{182167945}) / 333999126 \approx$ 10.5 , which is positive (since $492445959775^{2}=242503023298720918050625>$ $\left.(36433589 \sqrt{182167945})^{2}=241810897419701928577345\right)$.

Remark 2. The calculations in this final claim are remarkably tight. Numerical computations show that if we had broken the interval $(0,1)$ at a point $\gtrsim 0.602$, instead of $3 / 5$, then this argument would fail, so we are fortunate that $(9-\sqrt{61}) / 2 \approx 0.595$ is less than this. We also remark that uniqueness of solutions to these equations can fail if we allow $s_{1}, s_{2} \in(2 / 3,1)$, so the integrality conditions are crucial.

Depending on one's point of view, there are two possible responses to this serendipity. The first is that it is just a coincidence that we obtain unique WBF metrics in this (low-dimensional) situation. The second is that there is a general uniqueness theorem for WBF metrics. We leave it to the reader to decide.

\section{Acknowledgements}

The first author was supported by an NSERC Discovery Grant, the second author by an EPSRC Advanced Research Fellowship and the fourth author by the Union College Faculty Research Fund.

\section{References}

[1] V. Apostolov, D.M.J. Calderbank and P. Gauduchon, The geometry of weakly self-dual Kähler surfaces, Compositio Math. 135 (2003), $279-322$. 
[2] V. Apostolov, D.M.J. Calderbank and P. Gauduchon, Hamiltonian 2-forms in Kähler geometry I General theory, J. Differ. Geom. 73 (2006), 359-412.

[3] V. Apostolov, D.M.J. Calderbank, P. Gauduchon and C. TønnesenFriedman, Hamiltonian 2-forms in Kähler geometry II global classification, J. Differ. Geom. 68 (2004), 277-345.

[4] V. Apostolov, D.M.J. Calderbank, P. Gauduchon and C. Tønnesen-Friedman, Hamiltonian 2-forms in Kähler geometry III Extremal metrics and stability, Invent. Math. (2008, to appear), arXiv:math.DG/0511118.

[5] S. Bando and T. Mabuchi, Uniqueness of Einstein-Kähler metrics modulo connected group actions, in 'Algebraic Geometry' (Sendai 1985), Adv. Stud. Pure Math. 10, North Holland, Amsterdam, 1987, 11-40.

[6] R. Bryant, Bochner-Kähler metrics, J. Am. Math. Soc. 14 (2001), 623-715.

[7] E. Calabi, Extremal Kähler metrics, Seminar on differential geometry, Princeton University Press, 1982, 259-290.

[8] G. Elencwajg and M.S. Narasimhan, Projective bundles on a complex torus, J. Reine Angew. Math. 340 (1983), 1-5.

[9] A. Fujiki, Remarks on extremal Kähler metrics on ruled manifolds, Nagoya Math. J. 126 (1992), 89-101.

[10] S. Kobayashi and T. Ochiai, Characterization of complex projective spaces and hyperquadrics, J. Math. Kyoto Univ. 13 (1973), 31-47.

[11] N. Koiso, On rationally symmetric Hamilton's equations for KählerEinstein metrics, in 'Recent topics in differential and analytic geometry', Adv. Studies in Pure Math. 18-I, Academic Press, Boston, 1990, 327-337.

[12] N. Koiso and Y. Sakane, Nonhomogeneous Kähler-Einstein metrics on compact complex manifolds, in Curvature and topology of Riemannian manifolds (Kataka, 1985), Lecture Notes in Math. 1201, Springer, Berlin, 1986, 165-179.

[13] N. Koiso and Y. Sakane, Nonhomogeneous Kähler-Einstein metrics on compact complex manifolds II, Osaka J. Math. 25 (1988), 933-959. 
[14] M.S. Narasimhan and C.S. Seshadri, Stable and unitary vector bundles on a compact Riemann surface, Ann. Math. 82 (1965), 540-567.

[15] Y. Sakane, Examples of compact Einstein Kähler manifolds with positive Ricci tensor. Osaka J. Math. 23 (1986), 585-616.

[16] T. Suwa, Ruled surfaces of genus one, J. Math. Soc. Japan 2 (1969), 258-291.

[17] G. Tian On Calabi's conjecture for complex surfaces with positive first Chern class, Invent. Math. 101 (1990), 101-172.

[18] G. Tian, and X. Zhu, Uniqueness of Kähler-Ricci solitons, Acta Math. 184 (2000), 271-305.

[19] G. Tian and X. Zhu, A new holomorphic invariant and uniqueness of Kähler-Ricci solitons, Comment. Math. Helv. 77 (2002), 297-325.

[20] X.-J. Wang and X. Zhu, Kähler-Ricci solitons on toric manifolds with positive first Chern class. Adv. Math. 188 (2004), 87-103.

DÉPARTEMENT DE MATHÉMATIQUES

UQAM

C.P. 8888, Succ. Centre-ville

Montréal, (QuÉBEC)

Canada H3C 3P8

Institue OF MATHEMATICS AND INFORMATICS

Bulgarian ACADEMY of ScIEnCES

Acad. G. Bonchev Str. Bl. 8

1113 SOFIA

BULGARIA

E-mail address: apostolov.vestislav@uqam.ca

Department of Mathematics

UNIVERSITY OF YORK

Heslington, YoRK YO10 5DD

UK

Current ADDREss

Department of Mathematical Sciences

UNIVERSITY OF BATH

BATH BA2 7AY

UK

E-mail address: D.M.J.Calderbank@bath.ac.uk 
Centre de Mathématiques

École Polytechnique

UMR 7640 DU CNRS

91128 PALAisEAU

FRANCE

E-mail address: pg@math.polytechnique.fr

Department of Mathematics

Union College

SCHENECTADY

NY 12308

USA

E-mail address: tonnesec@union.edu

Received December 24, 2006 